\title{
TROPICAL SEVERI VARIETIES
}

\author{
JIHYEON JESSIE YANG
}

\begin{abstract}
We study the tropicalizations of Severi varieties, which we call tropical Severi varieties. In this paper, we give a partial answer to the following question, "describe the tropical Severi varieties explicitly." We obtain a description of tropical Severi varieties in terms of regular subdivisions of polygons. As an intermediate step, we construct explicit parameter spaces of curves. These parameter spaces are much simpler objects than the corresponding Severi variety and they are closely related to flat degenerations of the Severi variety, which in turn describes the tropical Severi variety. As an application, we understand G.Mikhalkin's correspondence theorem for the degrees of Severi varieties in terms of tropical intersection theory. In particular, this provides a proof of the independence of point-configurations in the enumeration of tropical nodal curves.
\end{abstract}

\section{INTRODUCTION}

The advent of tropical geometry and its fast development suggest to look at the classical algebraic geometry in a different perspective. Tropicalization is an operation that turns subvarieties of an algebraic torus into polyhedral objects in a real vector space along with a locally-constant integral-valued function on it. This procedure enables us to build an intersection theory on the algebraic torus called tropical intersection theory which can be used to solve classical enumerative questions. There are many attractive properties of this intersection theory. First of all, we work with polyhedral objects instead of algebraic varieties. Also we sometimes do not need to consider compactifications. The case of hypersurfaces is closely related to Newton polytope theory: the tropicalization of a hypersurface defined by a Laurent polynomial $f$ is the codimension 1 skeleton of the outer-normal fan of the Newton polytope of $f$. Moreover, the locally-constant integral-valued function on it is determined by the lattice lengths of the edges of the Newton polytope of $f$. Using tropical geometry, G. Mikhalkin[17] found a purely combinatorial method to compute the degree of a Severi variety (or Gromov-Witten invariants of plane $\mathbb{P}^{2}$ ). This celebrated work, correspondence theorem (Theorem 4.11), has brought tropical methods to the attention of geometers and motivated active systematic developments of tropical geometry. A Severi variety $\operatorname{Sev}(\Delta, \delta)$ (Definition 4.1) is a complex projective variety which parameterizes curves on the toric surface $X_{\Delta}$ of an integral polygon $\Delta$ with a given number $\delta$ of nodal singularities. It is known that the degree of a Severi variety is equal to the number of such nodal curves passing through a certain number of generic points in $\mathbb{C}^{2}$. Mikhalkin's correspondence theorem, simply speaking, says that this enumerative number is equal to the number of tropical plane curves passing through the same number of generic points in $\mathbb{R}^{2}$ counted with

We would like to thank the referees for careful reading of the manuscript and giving numerous helpful suggestions. 
certain multiplicities, called Mikhalkin's multiplicity (4.4). These tropical plane curves are tropicalizations of classical algebraic curves and they are purely combinatorial objects. Therefore, the enumerative problem becomes a purely combinatorial one. This approach brings up some questions such as, "why does this count give the solution for the original classical problem?" and "what is the meaning of such multiplicities assigned to tropical curves?". The answers for these questions are given in the proof of Mikhalkin's correspondence theorem [17] or Shustin's proof based on algebraic geometry [22]. However, their proofs on the independence of point-configuration relies on the well-known fact that the number of classical nodal curves does not depend on the position of points, which is a fact from classical intersection theory. Another proof of the independence of point-configuration is proved in 9 by considering moduli spaces of tropical curves.

In this paper, we take another approach: we study the tropicalization of a Severi variety $\operatorname{Trop}(\operatorname{Sev}(\Delta, \delta))$ and understand the enumeration of nodal curves in terms of tropical intersection theory. The subset $\operatorname{Trop}(\operatorname{Sev}(\Delta, \delta))$ of a real vector space has a natural weighted fan structure induced from the Gröbner fan of $\operatorname{Sev}(\Delta, \delta)$. The main ingredients of the definition of $\operatorname{Trop}(\operatorname{Sev}(\Delta, \delta))$ are the initial schemes $\operatorname{in}_{\omega} \operatorname{Sev}(\Delta, \delta)$ of the very affine Severi variety, $\operatorname{Sev}(\Delta, \delta)^{\circ}$, which is the intersection of the Severi variety $\operatorname{Sev}(\Delta, \delta)$ and the big open torus of the ambient projective space of $\operatorname{Sev}(\Delta, \delta)$. When $\omega$ is a regular point of $\operatorname{Trop}(\operatorname{Sev}(\Delta, \delta))$, that is, it is in the relative interior of a maximal cone of $\operatorname{Trop}(\operatorname{Sev}(\Delta, \delta))$, we know that the initial scheme $\operatorname{in}_{\omega} \operatorname{Sev}(\Delta, \delta)$ is supported on the union of finitely many translates of a subtorus of the big torus (for example, see [12, §6.]). The number of such translations of a subtorus (counted with multiplicities) is called the weight of $\omega$. The first simple description of (the support of) $\operatorname{Trop}(\operatorname{Sev}(\Delta, \delta))$ is given by a positive integer $\operatorname{rank}(\omega)$ (Definition 2.10) assigned to every point in the ambient real vector space of $\operatorname{Trop}(\operatorname{Sev}(\Delta, \delta))$ :

Theorem 1.1 (Theorem4.4. If an integral point $\omega$ has $\operatorname{rank}(\omega)>\operatorname{dim}(\operatorname{Sev}(\Delta, \delta))$, then $\omega$ is not in $\operatorname{Trop}(\operatorname{Sev}(\Delta, \delta))$.

The following two descriptions of $\operatorname{Trop}(\operatorname{Sev}(\Delta, \delta))$ uses the regular subdivisions of polygons $(\{2.3 .1$.

Theorem 1.2 (Theorem 4.6). Let $\omega$ be an integral point in $\operatorname{Trop}(\operatorname{Sev}(\Delta, \delta))$ with the following conditions:

- $\operatorname{rank}(\omega)=\operatorname{dim}(\operatorname{Sev}(\Delta, \delta))$;

- The regular subdivision $\Delta_{\omega}$ has no non-primitive parallelogram.

Then $\omega$ is a regular point of $\operatorname{Trop}(\operatorname{Sev}(\Delta, \delta))$, that is, $\omega$ is in a maximal cone of $\operatorname{Trop}(\operatorname{Sev}(\Delta, \delta))$. Furthermore, the weight of $\omega$ on $\operatorname{Trop}(\operatorname{Sev}(\Delta, \delta))$ is equal to

$$
\boldsymbol{m}_{\mathrm{Sev}(\Delta, \delta)}(\omega)=l(\mathbb{V}) \cdot \widetilde{\prod} \operatorname{length}\left(\operatorname{Edges}\left(\Delta_{\omega}\right)\right)
$$

where

(1) $l(\mathbb{V})$ is the number of connected components of $\mathbb{V}$;

(2) $\widetilde{\prod}$ length $\left(\operatorname{Edges}\left(\Delta_{\omega}\right)\right)$ is the product of the lattice lengths of the edges which are representatives of each equivalence class in $\operatorname{Edges}\left(\Delta_{\omega}\right)$, where we define an equivalence relation as follows: let $e \sim e^{\prime}$ if $e$ and $e^{\prime}$ are the parallel edges of a parallelogram in $\Delta_{\omega}$ and extend it by transitivity. 
Theorem 1.3 (Theorem 4.7). Let $\omega$ be an integral point in $\operatorname{Trop}(\operatorname{Sev}(\Delta, \delta))$ with the following conditions:

- $\operatorname{rank}(\omega)=\operatorname{dim}(\operatorname{Sev}(\Delta, \delta))$;

- $\omega$ is a regular point in $\operatorname{Trop}(\operatorname{Sev}(\Delta, \delta))$.

Then the weight of $\omega$ on $\operatorname{Trop}(\operatorname{Sev}(\Delta, \delta))$ is equal to

$$
\boldsymbol{m}_{\operatorname{Sev}(\Delta, \delta)}(\omega)=l(\mathbb{V}) \cdot \widetilde{\prod} \text { length }\left(\operatorname{Edges}\left(\Delta_{\omega}\right)\right),
$$

as defined in the previous theorem.

Using these results on tropical Severi varieties, we provide another proof of the independence of the configuration of points in the enumerations of tropical plane curves $(4.4)$.

\section{Preliminaries}

In tropical geometry, we study tropical varieties, which are polyhedral objects with certain properties. The precise definition is presented in $\$ 2.1$. Classical algebraic varieties are connected with tropical varieties by an operation called tropicalization. In this section, we review only what we need to study parameter spaces of algebraic curves on toric surfaces.

2.1. Tropical Varieties. 16 Let $\Sigma \subset \mathbb{R}^{n}$ be a one-dimensional fan with $r$ rays. Let $\boldsymbol{u}_{i}$ be the first lattice point on the $i$ th ray of $\Sigma$. We give $\Sigma$ the structure of a weighted fan by assigning a weight $m_{i} \in \mathbb{N}$ to the $i$ th ray of $\Sigma$. We say that $\Sigma$ is balanced if

$$
\sum_{i=1}^{r} m_{i} \boldsymbol{u}_{i}=0
$$

We now extend this concept to arbitrary polyhedral complexes.

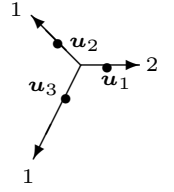

Definition 2.1. Let $\Sigma \subset \mathbb{R}^{n}$ be a rational polyhedral fan, of pure dimension $d$, and fix $m(\sigma) \in \mathbb{N}$ for all maximal cones $\sigma$. Such $\Sigma$ is called a weighted fan. We denote the set of all cones of dimension $k$ in $\Sigma$ by $\Sigma^{(d-k)}$. Let $\tau \in \Sigma^{(1)}$ and let $L_{\tau}$ be the affine span of $\tau$. Note that since $\tau$ is a rational cone, $L_{\mathbb{Z}}=L \cap \mathbb{Z}^{n}$ is a free abelian group of rank $d-1$ with $\mathbb{Z}^{n} / L_{\mathbb{Z}} \cong \mathbb{Z}^{n-d+1}$. For each maximal cone $\sigma \in \Sigma$ with $\tau \subset \sigma$ the cone $\left(\sigma+L_{\tau}\right) / L_{\tau}$ is a one-dimensional cone (ray) in $\mathbb{R}^{n} / L_{\tau}$. Let $\boldsymbol{u}_{\sigma / \tau}$ be the first lattice point on this ray. The weighted fan $\Sigma$ is balanced at $\tau$ if

$$
\sum m(\sigma) \boldsymbol{u}_{\sigma / \tau}=0 \in \mathbb{R}^{n} / L_{\tau},
$$

as the sum varies over all maximal cones containing $\tau$.

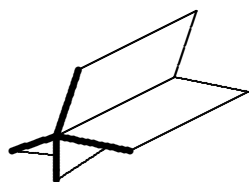

The weighted fan $\Sigma$ is balanced if it is balanced at all $\tau \in \Sigma^{(1)}$. Now let $\Sigma$ be a rational polyhedral complex of pure dimension $d$ with weight $m(\sigma) \in \mathbb{N}$ on each maximal polyhedron $\sigma$ in $\Sigma$. Then for each $\tau \in \Sigma$ the fan $\operatorname{star}_{\Sigma}(\tau)$ inherits a weighting function $m$, where $\operatorname{star}_{\Sigma}(\tau)$ is the star of $\tau \in \Sigma$ whose cones are indexed by those $\sigma \in \Sigma$ for which $\tau$ is a face of $\sigma$ : Fix $\omega \in \tau$. Then the cone of $\operatorname{star}_{\Sigma}(\tau)$ indexed by $\sigma$ is the Minkowski sum

$$
\bar{\sigma}:=\left\{v \in \mathbb{R}^{n}: \exists \epsilon>0 \text { with } \omega+\epsilon v \in \sigma\right\}+\operatorname{aff}(\tau)-\omega,
$$


where $\operatorname{aff}(\tau)$ is the affine span of $\tau$. This is independent of the choice of $\omega$. The

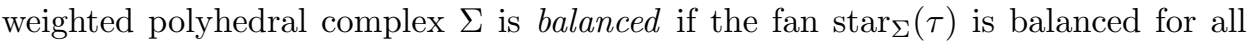
$\tau \in \Sigma^{(1)}$.

Definition 2.2. [1, [14, [24, §1]

(1) A homogeneous tropical variety of degree $n-d$ is a pair $(\mathcal{T}, \boldsymbol{m})$, where $\mathcal{T}$ is a subset of $\mathbb{R}^{n}$ and $\boldsymbol{m}: \mathcal{T}^{\circ} \rightarrow \mathbb{Z}_{>0}$ is a locally constant function, called weighting function which satisfies:

- There exists a pure $d$-dimensional rational polyhedral complex supported on $\mathcal{T}$;

- $\mathcal{T}^{\circ} \subset \mathcal{T}$ is the open subset of regular points, where $\omega \in \mathcal{T}$ is called regular if there exists a vector subspace $L_{\omega} \subset \mathbb{R}^{n}$ such that locally near $\omega, \mathcal{T}$ is equal to a translation $L_{\omega}+v$ of $L_{\omega}$ for some $v \in \mathbb{R}^{n}$.

- The function $\boldsymbol{m}$ satisfies the balancing condition for one (and hence for any) polyhedral complex supported on the set $\mathcal{T}$.

(2) A tropical cycle is a formal sum of homogeneous tropical varieties of different degrees.

2.2. Tropicalization. [12, [14, [16]

We consider an extension of the complex field $\mathbb{C}$ : Let $\mathbb{K}$ denote the field of locally convergent Puiseux series over $\mathbb{C}$, that is, the elements of $\mathbb{K}$ are power series of the form

$$
b(t)=\sum_{\tau \in R} c_{\tau} t^{\tau},
$$

where $R \subset \mathbb{Q}$ is contained in an arithmetic progression bounded from above, $c_{\tau} \in \mathbb{C}$, and $\sum_{\tau \in R}\left|c_{\tau}\right| t^{\tau}<\infty$ for sufficiently large positive $t$. This is an algebraically closed field of characteristic zero with a non-Archimedean valuation

$$
\operatorname{Val}(b)=\max \left\{\tau \in R: c_{\tau} \neq 0\right\} .
$$

Without loss of generality we may suppose that $\operatorname{Val}(b)$ is an integer by changing the parameter $t \mapsto t^{l}$ for some $l$. We always assume this unless mentioned otherwise. Note that this definition of $\mathbb{K}$ is slightly different from the one in 22 . However, the one in 22] can be obtained by the substitution, $t \mapsto t^{-1}$. Also the tropicalization can be defined for a general field with a non-Archimedean valuation. However, it is enough to consider only $\mathbb{K}$ for our purpose of the study of parameter spaces of curves on toric surfaces.

By a scheme we shall mean an algebraic scheme over the field $\mathbb{K}$, that is, a scheme $X$ together with a morphism of finite type from $X$ to $\operatorname{Spec}(\mathbb{K})$. A variety will be a reduced scheme, and a subvariety of a scheme will be a closed reduced subscheme. A point on a scheme will always be a closed point. A curve is a 1-dimensional scheme. In fact, the tropicalization is an operation which is defined only on subvarieties of an algebraic torus $\left(\mathbb{K}^{*}\right)^{n}=\operatorname{Spec}\left(\mathbb{K}\left[\mathbb{Z}^{n}\right]\right)$. The subvarieties of $\left(\mathbb{K}^{*}\right)^{n}$ are called very affine varieties. We often use the vector-notation, for example, $c^{a}$ is $c_{1}^{i} \cdot c_{2}^{j}$ and $c_{a}$ is $c_{(i, j)}$ where $a=(i, j)$. Also we often identify a Laurent polynomial with a function on a finite set as follows: let $f=\sum_{a \in \mathcal{A}} c_{a} x^{a}$ be a Laurent polynomial with variables $x$ and coefficients $c_{a}$ in a ring $\mathcal{R}$, where $\mathcal{A}$ is a finite subset of $\mathbb{Z}^{n}$. Then $f$ is identified with the function

$$
f: \mathcal{A} \rightarrow \mathcal{R}, \quad a \mapsto c_{a} .
$$


2.2.1. Tropicalization: Varieties over $\mathbb{C}$. In this section we present a precise definition of the tropicalization of $X$, where $X$ is a subvariety of an algebraic torus over $\mathbb{C}, \mathbb{T}=\operatorname{Spec}\left(\mathbb{C}\left[\mathbb{Z}^{n}\right]\right)=\operatorname{Spec}\left(\mathbb{C}\left[x_{1}^{ \pm 1}, \ldots, x_{n}^{ \pm 1}\right]\right)$.

\section{Definitions 2.3.}

- (initial sets) Given a finite subset $\mathcal{A}$ of $\mathbb{Z}^{n}$ and a vector $\omega \in \mathbb{Z}^{n}$, let $\operatorname{in}_{\omega} \mathcal{A}$ denote the set of all points $a \in \mathcal{A}$ such that the inner product $a \cdot \omega$ is maximal.

- (initial polynomials) Given a Laurent polynomial $f \in\left(\mathbb{C}^{*}\right)^{\mathcal{A}}$ and a vector $\omega \in \mathbb{Z}^{n}$, the $\omega$-degree of $f$ is the maximum of $a \cdot \omega$ for all $a \in \mathcal{A}$. Let $\operatorname{in}_{\omega} f$ denote the restriction of $f$ to $\operatorname{in}_{\omega} \mathcal{A}$ so that $\operatorname{in}_{\omega} f$ is homogeneous with respect to $\omega$-degree. We can also see that

$$
\operatorname{in}_{\omega} f(x)=\lim _{t \rightarrow \infty} t^{-\gamma} f\left(t^{\omega} \cdot x\right),
$$

where $\gamma$ is the $\omega$-degree of $f$.

- (initial ideals) Given an ideal $I$ in the ring of Laurent polynomials $\mathbb{C}\left[\mathbb{Z}^{n}\right]$ and a vector $\omega \in \mathbb{Z}^{n}$, let $\operatorname{in}_{\omega} I$ denote the ideal generated by all initial polynomials $\operatorname{in}_{\omega} f$ for $f \in I$.

- (initial schemes) Given an affine scheme $V(I):=\operatorname{Spec}\left(\mathbb{C}\left[\mathbb{Z}^{n}\right] / I\right)$ and a vector $\omega \in \mathbb{Z}^{n}$, the scheme $V\left(\operatorname{in}_{\omega} I\right):=\operatorname{Spec}\left(\mathbb{C}\left[\mathbb{Z}^{n}\right] / \operatorname{in}_{\omega} I\right)$ defined by $\operatorname{in}_{\omega} I$ is called the initial scheme of $V(I)$ with respect to $\omega$.

Remark 2.4. We may consider the initial scheme $V\left(\operatorname{in}_{\omega} I\right)$ as a flat degeneration of $V(I)$, that is, there exist a one-parameter flat family $V\left(I_{t}\right)$ such that $V(I)=V\left(I_{1}\right)$ and $V\left(\operatorname{in}_{\omega} I\right)=V\left(I_{0}\right)$. The details are given in [5, $\left.\S 15.8\right]$.

Definitions 2.5. Let $X$ be an irreducible subvariety of the algebraic torus $\mathbb{T}=\mathbb{T}_{\mathbb{C}}$.

- A vector $\omega \in \mathbb{Z}^{n}$ is called a c-vector of $X$ if the initial ideal $\operatorname{in}_{\omega} I_{X}$ of the defining ideal $I_{X}$ of $X$ contains no monomial, equivalently, the initial scheme $\operatorname{in}_{\omega} X:=V\left(\operatorname{in}_{\omega} I_{X}\right)$ is not empty. ("c" is the first letter of "current" as introduced in 14])

- The (support of the) tropicalization of $X$ is the closure of the union of positive rays $\mathbb{R}_{\geq 0} \cdot \omega$ generated by all c-vectors $\omega$ of $X$ and it is denoted by $\operatorname{Trop}(X)$.

- We define a weighting function $\boldsymbol{m}_{X}$ on $\operatorname{Trop}(X)$ as follows: For a point $\omega$ in $\operatorname{Trop}(X)$ let $\boldsymbol{m}(\omega)$ to be the sum of the multiplicities of all minimal associated prime ideals of the initial ideal $\operatorname{in}_{\omega} I_{X}$.

- The set $\operatorname{Trop}(X)$ together with the weighting function $\boldsymbol{m}_{X}$ is called the tropicalization of $X$ and it is denoted again by $\operatorname{Trop}(X)$.

Example 2.6. Let $X=V\left((1+x+y)^{2}\right)$ be the plane curve. The picture below is the support of $\operatorname{Trop}(X)$. The integers near the three rays are the corresponding weights. For example, let $\omega=(0,-1)$. The corresponding initial polynomial, $\operatorname{in}_{(0,-1)} f$, is equal to $(1+x)^{2}$ and so $\boldsymbol{m}((0,-1))=2$.

2.2.2. Tropicalization. Now we consider the general case: varieties over $\mathbb{K} \supset \mathbb{C}$. As noted in Remark 2.4 for varieties defined over $\mathbb{C}$, tropicalization is a way to record certain flat limits. Geometrically, the extension of field from $\mathbb{C}$ to $\mathbb{K}$ can be seen as adding one parameter $t$ and considering flat families of varieties. This idea is well described in Proposition 2.8 in the case of curves on toric surfaces. By generalizing 
the definition of the initial ideal to consider the role of the new parameter $t$, we can define the tropicalization of a variety over $\mathbb{K}$ in a straightforward way.

\section{Definitions 2.7.}

- (initial sets) Let $(\mathcal{A}, \nu)$ be a pair of a finite subset $\mathcal{A}$ of $\mathbb{Z}^{n}$ and a real-valued function $\nu$ on it. Given a $(\mathcal{A}, \nu)$ and a vector $\omega$ in $\mathbb{Z}^{n}$, let in $\operatorname{in}_{\omega}(\mathcal{A}, \nu)$ denote the set of all points $a \in \mathcal{A}$ such that $a \cdot \omega+\nu(a)$ is maximal.

- (initial polynomials) Given a Laurent polynomial $f=\sum_{a \in \mathcal{A}} c_{a}(t) x^{a}$ over $\mathbb{K}$ and a vector $\omega \in \mathbb{Z}^{n}$, the $t-\omega$-degree of $f$ is the maximum of $a \cdot \omega+\operatorname{Val}\left(c_{a}\right)$ for all $a \in \mathcal{A}$. We define $\operatorname{in}_{\omega} f$ which is defined over $\mathbb{C}$ as follows:

$$
\operatorname{in}_{\omega} f(x)=\lim _{t \rightarrow \infty} t^{-\gamma} f\left(t^{\omega} \cdot x\right),
$$

where $\gamma$ is the $t-\omega$-degree of $f$. The following shows how to obtain $\operatorname{in}_{\omega} f$ : Let $\Delta=\operatorname{Newton}(f)$ be the Newton polytope of $f$. We take the convex hull $\widetilde{\Delta}$ of the set $\left\{\left(a, \operatorname{Val}\left(c_{a}\right)\right) \in \mathbb{Z}^{n+1}: a \in \mathcal{A}\right\}$ and introduce the function

$$
\nu_{f}: \Delta \rightarrow \mathbb{R}, \quad \nu_{f}(\alpha)=\max \{\beta:(\alpha, \beta) \in \widetilde{\Delta}\} .
$$

This is a concave piecewise-linear function. Notice that we can write

$$
c_{a}(t)=c_{a}^{\circ} t^{\nu_{f}(a)}+\text { l.o.t. },
$$

where $c_{a}^{\circ}$ is some complex number which is zero if $\nu_{f}(a)>\operatorname{Val}\left(c_{a}\right)$. Then

$$
\operatorname{in}_{\omega} f=\sum_{a \in \operatorname{in}_{\omega}\left(\mathcal{A}, \nu_{f}\right)} c_{a}^{\circ} x^{a}
$$

- (initial ideals) For an ideal $I$ in the ring of Laurent polynomials $\mathbb{K}\left[\mathbb{Z}^{n}\right]$ and a vector $\omega \in \mathbb{Z}^{n}$, let $\operatorname{in}_{\omega} I \subset \mathbb{C}\left[\mathbb{Z}^{n}\right]$ denote the ideal generated by all initial polynomials $\operatorname{in}_{\omega} f$ for $f \in I$.

The definition of the tropicalization given in the previous subsection 2.2 .1 generalizes in a straightforward way for an irreducible subvariety $X$ of the algebraic torus $\mathbb{T}_{\mathbb{K}}$.

Now the proofs of the following facts can be found in many references on tropical geometry, for example, [3], 4, [16]:

- The tropicalization $\operatorname{Trop}(X)$ has the structure of a homogeneous tropical variety with dimension $\operatorname{dim}(X)$

- Suppose $X$ is defined over $\mathbb{C}$, i,e., the ideal $I_{X} \subset \mathbb{K}\left[\mathbb{Z}^{n}\right]$ of $X$ can be generated by Laurent polynomials over $\mathbb{C}$ and thus $X$ is independent of the parameter $t$. (Sometimes, this case is called constant coefficient case.) Then $\operatorname{Trop}(X)$ coincides with $\operatorname{Trop}(X(\mathbb{C}))$, that is, we can treat it as in the previous section. Furthermore, in this case $\left(\operatorname{Trop}(X), \boldsymbol{m}_{X}\right)$ has a balanced polyhedral fan structure.

- For any regular point $\omega \in \operatorname{Trop}(X)$, the initial scheme $\operatorname{in}_{\omega} X$ is a union of finitely many translations of a subtorus $\mathbb{G}^{e}$ of $\mathbb{T}=\mathbb{T}_{\mathbb{C}}$.

2.3. Tropical plane curves. We want to study complex algebraic curves on toric surfaces by studying their tropicalizations. Recall that tropicalization is defined for subvarieties of algebraic tori. Thus we only consider the intersections of the curves with the big open torus $\left(\mathbb{C}^{*}\right)^{2}$ of the toric surfaces. It is very easy to understand the tropicalizations of the curves: any such curve $X$ in $\left(\mathbb{C}^{*}\right)^{2}$ is defined by a Laurent polynomial $f(x, y)$ in two variables over $\mathbb{C}$. The tropicalization $\operatorname{Trop}(X)$ is the 
1-dimensional skeleton of the (outer) normal fan of the Newton polygon of $f$ and the multiplicity of each ray is the lattice length of the corresponding edge of the Newton polygon of $f$ (see Example 2.6). The problem is that $\operatorname{Trop}(X)$ does not distinguish much about the curves because curves with the same Newton polygon have the same tropicalization. This lack of information is overcome by considering a more generalized version of $\operatorname{Trop}(X)$. Namely, we extend the field of coefficients from $\mathbb{C}$ to $\mathbb{K}$, the field of locally convergent Puiseux series over $\mathbb{C}$. The relationship between algebraic curves over $\mathbb{C}$ and $\mathbb{K}$ is formulated in the following statement.

Proposition 2.8. [22, §2.3.] Let a Laurent polynomial

$$
f(x, y)=\sum_{(i, j) \in \Delta} c_{i j}(t) x^{i} y^{j} \in \mathbb{K}[x, y]
$$

define a curve $C_{\mathbb{K}} \subset\left(\mathbb{K}^{*}\right)^{2}$ with only isolated singularities and Newton polygon $\Delta$. We obtain a one-parameter family $C^{(t)}$ of curves on $\left(\mathbb{C}^{*}\right)^{2}$. For $t$ with $|t|>>0$, the family $C^{(t)}$ is equisingular and the topological types of singularities of $C^{(t)}$ are in 1-to-1 correspondence with topological types of singularities of $C_{\mathbb{K}}$.

Shustin [22] found nice characterizations of the tropicalizations of nodal curves on toric surfaces which are crucial to the study of the initial schemes of Severi varieties. However, these characterizations are valid only for nodal curves satisfying certain base-point conditions. In the following subsections we show that how we can replace the base-point conditions by the rank-condition (Definition 2.10).

\subsubsection{Subdivisions of Polygons and Adjacency graphs.}

Definitions 2.9. Let $\Delta$ be a convex lattice polygon.

(1) A subdivision of $\Delta$, denoted by $\mathcal{S}(\Delta)$, is a decomposition of $\Delta$ into a finite number of non-degenerate convex lattice sub-polygons such that the intersection of any two of these sub-polygons is a common face of both of them (maybe empty). (We consider $\Delta$ itself as a subdivision of $\Delta$ with one two-dimensional face.)

(2) Given a subdivision $\mathcal{S}(\Delta)$, let $\operatorname{Vertices}(\mathcal{S}(\Delta))$, $\operatorname{Edges}(\mathcal{S}(\Delta))$, Faces $(\mathcal{S}(\Delta))$, Triangles $(\mathcal{S}(\Delta))$, Parallelograms $(\mathcal{S}(\Delta)), \operatorname{Int}(\mathcal{S}(\Delta)) \cap \mathbb{Z}^{2}$ be the set of vertices, edges, (2-dimensional) faces, triangles, parallelograms , interior lattice points of $\mathcal{S}(\Delta)$, respectively.

(3) A subdivision $\mathcal{S}(\Delta)$ is called

- triangular if every 2-dimensional face is a triangle;

- nodal if every 2-dimensional face is either a triangle or a parallelogram;

- simple if every lattice point on the boundary of $\Delta$ is a vertex of $\mathcal{S}(\Delta)$.

(4) A subdivision $\mathcal{S}(\Delta)$ is called regular if there exists a continuous concave piecewise-linear function on $\Delta$ whose domains of linearity are precisely the 2-dimensional faces of $\mathcal{S}(\Delta)$.

(5) Given a regular subdivision $\mathcal{S}(\Delta)$, consider the set of all concave piecewiselinear functions on $\Delta$ whose domains of linearity induce the subdivision $\mathcal{S}(\Delta)$. As embedded in $\mathbb{R}^{\Delta \cap \mathbb{Z}^{2}}$, this set is a polyhedral cone. We take its image in the quotient space $\mathbb{R}^{\Delta \cap \mathbb{Z}^{2}} / \mathbb{R} \cdot(1, \ldots, 1)$ which is again a polyhedral cone and denote it by $\mathcal{T} C(\mathcal{S}(\Delta))$, called the tropical cone of $\mathcal{S}(\Delta)$. Its dimension is called the rank of $\mathcal{S}(\Delta)$ and denoted by $\operatorname{rank}(\mathcal{S}(\Delta))$. 
Now let $\psi: \Delta \cap \mathbb{Z}^{2} \rightarrow \mathbb{R}$ be a real-valued function defined on $\Delta \cap \mathbb{Z}^{2}$. We construct a regular subdivision $\Delta_{\psi}$ of $\Delta$ from $\psi$ as follows:

Let $G_{\psi} \subset \mathbb{R}^{3}$ be the convex hull of the set

$$
\left\{(a, y): \quad y \leq \psi(a), \quad a \in \Delta \cap \mathbb{Z}^{2}\right\} .
$$

Then the upper boundary of $G_{\psi}$ is the graph of a concave piecewise-linear function $c c(\psi)$ which is called the concave hull of $\psi$. (The upper boundary of $G_{\psi}$ is by definition the union of faces of $G_{\psi}$ which do not contain vertical half-lines.) Let $\Delta_{\psi}$ denote the regular subdivision of $\Delta$ given by the domains of linearity of $c c(\psi)$. The figure on the right illustrates the case when $\Delta$ is the segment, $\operatorname{conv}(0, \ldots, 4)$. The subdivision $\Delta_{\psi}$ is the union of two segments $\operatorname{conv}(0,2)$ and $\operatorname{conv}(2,4)$ (We denote the convex hull of a set $A$ by $\downarrow \checkmark G_{\psi}$ $\operatorname{conv}(A)$.)

Let us consider the tropical cone $\mathcal{T} C(\psi):=\mathcal{T} C\left(\Delta_{\psi}\right)$. It contains the concave hull of $\psi$ restricted on $\Delta \cap \mathbb{Z}^{2}:\left.c c(\psi)\right|_{\Delta \cap \mathbb{Z}^{2}} \in \mathcal{T} C(\psi)$. Notice that $\left.c c(\psi)\right|_{\Delta \cap \mathbb{Z}^{2}}$ may not coincide with $\psi$ and in such case $\psi \notin \mathcal{T} C(\psi)$.

Definition 2.10. The rank of $\psi$, written as $\operatorname{rank}(\psi)$, is the rank of the regular subdivision $\Delta_{\psi}$.

Proposition 2.11. Suppose that $\mathcal{S}(\Delta)=\Delta_{\psi}$ is a regular nodal subdivision of $\Delta$. Then,

$$
\operatorname{rank}(\psi)=|\operatorname{Vertices}(\mathcal{S}(\Delta))|-1-|\operatorname{Parallelograms}(\mathcal{S}(\Delta))| .
$$

The proof of this Proposition can be easily deduced from [11, Lemma 2.40.].

Remark 2.12. The regular subdivisions of a lattice polytope is studied in [10, Ch.7] in which the secondary fan is introduced. In a forthcoming paper, the connection of the tropical cone $\mathcal{T} C(\psi)$ and $\operatorname{rank}(\psi)$ to the secondary fan will be studied.

Now we study the adjacency graph $\mathcal{S}(\Delta)^{*}$ of a given subdivision $\mathcal{S}(\Delta)$ of $\Delta$. By definition, the vertices $F^{*}$ of $\mathcal{S}(\Delta)^{*}$ correspond to the 2-dimensional faces $F$ of $\mathcal{S}(\Delta)$ and two vertices $F_{1}^{*}$ and $F_{2}^{*}$ of $\mathcal{S}(\Delta)^{*}$ are connected by an edge $\left(F_{1}^{*}, F_{2}^{*}\right)$ if the corresponding faces $F_{1}$ and $F_{2}$ of $\mathcal{S}(\Delta)$ have a common edge $\left(F_{1}, F_{2}\right)$ in $\mathcal{S}(\Delta)$. Given an orientation $\Gamma$ on $\operatorname{Edges}(\mathcal{S}(\Delta))$, we define an orientation $\Gamma^{*}$ on $\operatorname{Edges}\left(\mathcal{S}(\Delta)^{*}\right)$ as follows: direct $F_{1}^{*} \rightarrow F_{2}^{*}$, if the oriented edge $\left(F_{1}, F_{2}\right)$ and a normal vector to it leaving from $F_{1}$ to $F_{2}$ are positively oriented. Otherwise, direct $F_{2}^{*} \rightarrow F_{1}^{*}$. For any subdivision $\mathcal{S}(\Delta)$ of $\Delta$, we can always find an orientation $\Gamma$ on $\operatorname{Edges}(\mathcal{S}(\Delta))$ such that $\Gamma^{*}$ has neither an oriented cycle nor a sink. (A sink (resp. source) is a vertex $v$ such that all edges adjacent to $v$ are coming into (resp. leaving from) $v$.) In fact, an oriented cycle in $\Gamma^{*}$ corresponds to a sink or a source at an internal vertex of $(\mathcal{S}(\Delta), \Gamma)$. Also, a sink in $\Gamma^{*}$ corresponds to an oriented cycle in $\Gamma$. We can choose an orientation $\Gamma$ on $\operatorname{Edges}(\mathcal{S}(\Delta))$ such that $\Gamma$ has no oriented cycle and it also has no sink/source at an internal vertex of $\mathcal{S}(\Delta)$. For example, choose a generic vector $\zeta \in \mathbb{R}^{2} \backslash\{0\}$ and orient the edges of $\mathcal{S}(\Delta)$ so that they form acute angles with $\zeta$.
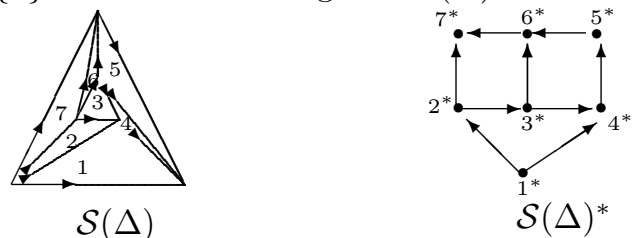

$\mathcal{S}(\Delta)$ 
2.3.2. Shustin's characterizations. Let $V_{\mathbb{K}}(f)$ be a curve defined by a Laurent polynomial over $\mathbb{K}$,

$$
f=\sum_{a \in \Delta \cap \mathbb{Z}^{2}} c_{a}(t) x^{a} \in \mathbb{K}\left[\mathbb{Z}^{2}\right], \quad c_{a} \in \mathbb{K} \backslash\{0\},
$$

where $\Delta=\operatorname{Newton}(f)$. The support of the tropicalization of $V_{\mathbb{K}}(f)$ is the corner locus of the piecewise-linear function

$$
\mathbb{R}^{2} \rightarrow \mathbb{R}, \quad \alpha \mapsto \max _{a \in \Delta \cap \mathbb{Z}^{2}}\left\{a \cdot \alpha+\operatorname{Val}_{f}(a)\right\},
$$

where $\mathrm{Val}_{f}$ is the function

$$
\operatorname{Val}_{f}: \Delta \cap \mathbb{Z}^{2} \rightarrow \mathbb{Z}, \quad a \mapsto \operatorname{Val}\left(c_{a}(t)\right) .
$$

Let us denote the support of the tropicalization of the curve $V_{\mathbb{K}}(f)$ by $\tau_{f}$. In general, the tropical curve $\tau_{\omega}$ is by definition the corner locus of the piecewise-linear function

$$
\mathbb{R}^{2} \rightarrow \mathbb{R}, \quad \alpha \mapsto \max _{a \in \Delta \cap \mathbb{Z}^{2}}\{a \cdot \alpha+\omega(a)\},
$$

where $\omega: \Delta \cap \mathbb{Z}^{2} \rightarrow \mathbb{Z}$ is an integral-valued function on $\Delta \cap \mathbb{Z}^{2}$. The regular subdivision $\Delta_{\omega}$ of $\Delta$ is dual to the tropical curve $\tau_{\omega}$ in the following sense (11. $\S 2.5 .1])$ :

- the components of $\mathbb{R}^{2} \backslash \tau_{\omega}$ are in 1-to-1 correspondence with $\operatorname{Vertices}\left(\Delta_{\omega}\right)$;

- the edges of $\tau_{\omega}$ are in 1-to-1 correspondence with $\operatorname{Edges}\left(\Delta_{\omega}\right)$ so that an edge $e$ of $\tau_{\omega}$ is dual to an edge of $\Delta_{\omega}$ which is orthogonal to $e$ with the lattice length $\omega(e)$;

- the vertices of $\tau_{\omega}$ are in 1-to-1 correspondence with the 2-dimensional faces of $\Delta_{\omega}$ so that the valency of a vertex of $\tau_{\omega}$ is equal to the number of sides of the dual face.

We call a tropical curve $\tau_{\omega}$ to be simple (respectively, triangular, nodal) if the dual regular subdivision $\Delta_{\omega}$ of $\Delta$ is simple (respectively, triangular, nodal). The rank of $\tau_{\omega}$ is by definition the rank of $\Delta_{\omega}$.

Example 2.13. Let $f=(1+x+y)(1+t x+t y)$. The picture below on the right is $\tau_{f}$ and one on the left is the corresponding subdivision of the Newton polygon of $f$.

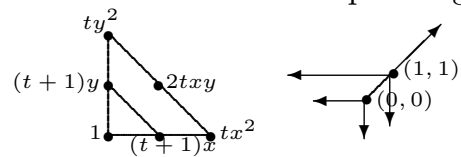

Now let us study the initial schemes of the curve $V_{\mathbb{K}}(f)$, which are defined by the initial polynomials of $f$. For this, we rewrite the coefficients of $f$, $c_{a}(t)=\overline{c_{a}} t^{V a l_{f}(a)}+$ l.o.t., $\quad \overline{c_{a}} \in(\mathbb{C})^{*}, \quad a \in \Delta \cap \mathbb{Z}^{2}$ as follows:

$$
c_{a}(t)=c_{a}^{\circ} t^{\nu_{f}(a)}+\text { l.o.t. }
$$

where $\nu_{f}$ is the concave hull of $\operatorname{Val}_{f}$ (defined as in 2.3.1) and $c_{a}^{\circ}$ is some complex number which is zero if $\nu_{f}(a)>\operatorname{Val}_{f}(a)$. Given a point $\alpha$ which is either a vertex or a point in the relative interior of an edge of $\tau_{f}$, the maximum of $a \cdot \alpha+\nu_{f}(a) \quad(a \in$ $\left.\Delta \cap \mathbb{Z}^{2}\right)$ is attained on the corresponding dual face or edge in $\Delta_{f}:=\Delta_{\nu_{f}}$. Therefore,

$$
\operatorname{in}_{\alpha} f=\sum c_{a}^{\circ} x^{a},
$$

where the sum runs over the lattice points on the dual face or edge in $\Delta_{f}$. In particular, we consider the initial polynomials $\operatorname{in}_{\alpha_{i}} f$, where $\alpha_{i}$ are the vertices of $\tau_{f}$ corresponding to the faces (2-dimensional subpolygons) $\Delta_{i}$ in $\Delta_{f}: \Delta_{1} \cup \cdots \cup \Delta_{m}$. 
Then, given a curve $V_{\mathbb{K}}(f)$ in $\left(\mathbb{K}^{*}\right)^{2}$ with $\operatorname{Newton}(f)=\Delta$ we obtain a collection of complex curves $V\left(\operatorname{in}_{\alpha_{i}} f\right)$ in $\left(\mathbb{C}^{*}\right)^{2}$ with Newton $\left(\operatorname{in}_{\alpha_{i}} f\right)=\Delta_{i}, \quad(i=1, \ldots, m)$. This collection of complex polynomials together with the subdivision $\Delta_{f}$ completely determines the tropicalization of the curve $V_{\mathbb{K}}(f)$ (or of $f$ ).

Now we can present Shustin's characterizations of the tropicalizations of nodal curves with a given rank condition:

Theorem 2.14. 22, §3.3] Let $V_{\mathbb{K}}(f)$ be a curve with $\delta$ nodal singular points (i.e. ordinary double points) as the only singularities, where $\delta$ is a natural number with $\delta \leq\left|\operatorname{Int}(\Delta) \cap \mathbb{Z}^{2}\right|$. Suppose that $\operatorname{rank}\left(\Delta_{f}\right) \geq r$, where $r=\left|\Delta \cap \mathbb{Z}^{2}\right|-1-\delta$. Then the following holds true:

(1) (combinatorial) the regular subdivision $\Delta_{f}: \Delta_{1} \cup \cdots \cup \Delta_{m}$ is simple, nodal, and $\operatorname{rank}\left(\Delta_{f}\right)=r$;

(2) (geometric)

- for each triangle $\Delta_{i}$, the curve $V\left(\operatorname{in}_{\alpha_{i}} f\right)$ is rational and meets the union of toric divisors $\operatorname{Tor}\left(\partial \Delta_{i}\right)$ at exactly three points, where it is unibranch;

- for each parallelogram $\Delta_{j}$, the polynomial $\operatorname{in}_{\alpha_{j}} f$ has the form

$$
\begin{gathered}
x^{k} y^{l}\left(\alpha x^{a}+\beta y^{b}\right)^{p}\left(\gamma x^{c}+\delta y^{d}\right)^{q} \\
\text { with }(a, b)=(c, d)=1,(a: b) \neq(c: d), \alpha, \beta, \gamma, \delta \in \mathbb{C} \backslash\{0\}
\end{gathered}
$$

Shustin's original statement $([22, \S 3.3])$ has the following base-point condition instead of the rank condition, $\operatorname{rank}\left(\Delta_{f}\right) \geq r$ : Suppose that the curve $V_{\mathbb{K}}(f)$ passes through $r$ generic points $p_{1}, \ldots, p_{r} \in\left(\mathbb{K}^{*}\right)^{2}$ such that $q_{1}=\operatorname{Val}\left(p_{1}\right), \ldots, q_{r}=$ $\operatorname{Val}\left(p_{r}\right) \in \mathbb{R}^{2}$ (taking valuations coordinatewise) are generic points (Definition 4.9), where $r=\left|\Delta \cap \mathbb{Z}^{2}\right|-1-\delta$.

We explain why we can replace the base-point condition by the rank condition: His proof is by estimating $\check{\chi}\left(C^{(t)}\right)$, the topological Euler characteristic of the normalization of the complex curve $C^{(t)}=V\left(f_{t}\right)$ (see Proposition 2.8) from above and from below and comparing the bounds. The upper bound of $\check{\chi}\left(C^{(t)}\right)$ in his computation holds true for any flat deformation. For a lower bound, he uses the following inequality:

$$
\begin{aligned}
\check{\chi}\left(C^{(t)}\right) & =2-2 g\left(C^{(t)}\right) \\
& =2-2\left(\left|\operatorname{Int}(\Delta) \cap \mathbb{Z}^{2}\right|-\delta\right) \\
& =2-2\left|\operatorname{Int}(\Delta) \cap \mathbb{Z}^{2}\right|+2\left(\left|\Delta \cap \mathbb{Z}^{2}\right|-1-r\right) \\
& =2\left|\partial \Delta \cap \mathbb{Z}^{2}\right|-2 r \\
& \geq 2\left|\partial \Delta \cap \mathbb{Z}^{2}\right|-2 \operatorname{rank}\left(\Delta_{f}\right)
\end{aligned}
$$

In the second equality, he used the fact that $C^{(t)}$ has $\delta$ nodal points. The last inequality follows from the condition, $r \leq \operatorname{rank}\left(\Delta_{f}\right)$. In fact, Shustin called the points $q_{1}, \ldots, g_{r}$ to be generic when $r \leq \operatorname{rank}\left(\Delta_{f}\right)$. (Definition 4.9 )

\subsection{Tropical Intersection Theory. 11, 12, 14, 15]}

Tropical cycles form a graded commutative algebra $\mathbf{A}$. To a subvariety $X$ of the algebraic torus $\mathbb{T}_{\mathbb{K}}=\operatorname{Spec}\left(\mathbb{K}\left[\mathbb{Z}^{n}\right]\right)$, we can assign an element $\operatorname{Trop}(X)$ of $\mathbf{A}$, namely the tropicalization of $X$. This correspondence determines an intersection theory of subvarieties of $\mathbb{T}_{\mathbb{K}}$. In this paper, we only summarize about the product $\mathcal{T}_{1} \cdot \mathcal{T}_{2}$ when $\mathcal{T}_{1}$ and $\mathcal{T}_{2}$ are complementary dimensional tropical varieties in $\mathbb{R}^{n}$. The support of the product $\mathcal{T}_{1} \cdot \mathcal{T}_{2}$ is by definition the zero-dimensional strata of the set 
$\operatorname{supp}\left(\mathcal{T}_{1}\right) \cap \operatorname{supp}\left(\mathcal{T}_{2}\right)$. It is a finite set of points in $\mathbb{R}^{n}$. The weighting function $\boldsymbol{m}=$ $\boldsymbol{m}_{\mathcal{T}_{1} \cdot \mathcal{T}_{2}}$ in the product $\mathcal{T}_{1} \cdot \mathcal{T}_{2}$ is defined as follows: let $\omega \in \operatorname{supp}\left(\mathcal{T}_{1}\right) \cap \operatorname{supp}\left(\mathcal{T}_{2}\right)$. There are two possible cases that $\omega$ is the intersection point of a transversal intersection or not.
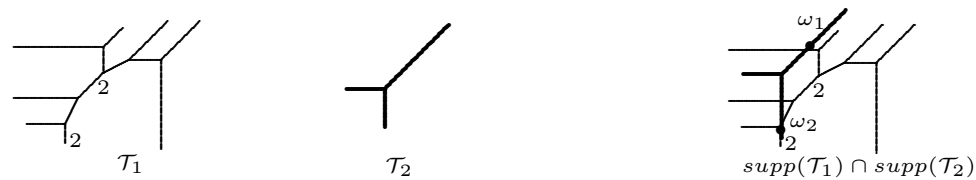

In the first case, $\omega$ is a regular point of each $\mathcal{T}_{i}$ and so $\mathcal{T}_{i}$ is equal to $L_{i}$ locally near $\omega, i=1,2$, where $L_{1}$ and $L_{2}$ are affine spaces of complementary dimensional.

(1) The extrinsic intersection multiplicity of $\mathcal{T}_{1}$ and $\mathcal{T}_{2}$ at $\omega$, denoted by $\xi\left(\omega ; \mathcal{T}_{1}, \mathcal{T}_{2}\right)$, is the volume of the parallelepiped constructed by the fundamental cells of the lattices $\mathbb{L}_{i} \cap \mathbb{Z}^{n},(i=1,2)$ ("principal parallelepiped")

(2) The tropical intersection multiplicity of $\mathcal{T}_{1}$ and $\mathcal{T}_{2}$ at $\omega$ is

$$
\boldsymbol{m}(\omega)=\boldsymbol{m}\left(\omega ; \mathcal{T}_{1}, \mathcal{T}_{2}\right):=\boldsymbol{m}_{\mathcal{T}_{1}}(\omega) \cdot \boldsymbol{m}_{\mathcal{T}_{2}}(\omega) \cdot \xi\left(\omega ; \mathcal{T}_{1}, \mathcal{T}_{2}\right)
$$

In the second case, $\omega$ is not a regular point of either $\mathcal{T}_{1}$ or $\mathcal{T}_{2}$. However, by a small local displacement of $\mathcal{T}_{1}$ and $\mathcal{T}_{2}$, we can achieve the transversality near $\omega$. (The details can be found in [8, [18]). Then $\boldsymbol{m}(\omega)$ is by definition the sum of all $\boldsymbol{m}(\tilde{\omega})$, where $\tilde{\omega}$ 's are the transversal intersection points appearing in the displacement.

When $\mathcal{T}_{i}=\operatorname{Trop}\left(X_{i}\right)$ for subvarieties $X_{i}$ of $\mathbb{T}_{\mathbb{K}}(i=1,2)$, the sum of weights on the product $\mathcal{T}_{1} \cdot \mathcal{T}_{2}$ is equal to the number of intersection points in $X_{1} \cap g X_{2}$ for a generic $g \in \mathbb{T}_{\mathbb{K}}$. It is called the degree of the product $\mathcal{T}_{1} \cdot \mathcal{T}_{2}$ and denoted by

$$
\left(\operatorname{Trop}\left(X_{1}\right) \cdot \operatorname{Trop}\left(X_{2}\right)\right) \text {. }
$$

For example,

- the degree of the product of tropicalizations of $n$ hypersurfaces in $\mathbb{T}_{\mathbb{C}}$ is the mixed volume of the Newton polyhedra of the hypersurfaces times $n$ !; (compare [2].)

- let $\mathbb{T}_{1}$ and $\mathbb{T}_{2}$ be two subtori of $\mathbb{T}_{\mathbb{C}}$ of complementary dimension. Then their tropicalizations are rational linear subspaces of $\mathbb{R}^{n}$ with constant multiplicity 1 and they are of complementary dimension. The degree of the product $\operatorname{Trop}\left(\mathbb{T}_{1}\right) \cdot \operatorname{Trop}\left(\mathbb{T}_{2}\right)$ is equal to the (normalized) volume of the parallelepiped defined by the fundamental cells of the lattices, $\operatorname{Trop}\left(\mathbb{T}_{1}\right) \cap \mathbb{Z}^{n}$ and $\operatorname{Trop}\left(\mathbb{T}_{2}\right) \cap \mathbb{Z}^{n}$.

\section{Intermediate PARAMETER SPACES}

In this section, we study certain parameter spaces of curves on toric surfaces, which are closely related to the initial schemes of Severi varieties. They are defined by considering Shustin's characterization (Theorem 2.14 in the view of parameter spaces and turn out to be very simple.

Let us consider the projective toric surface $X_{\Delta}$ constructed from a 2-dimensional lattice polygon $\Delta$ in $\mathbb{R}^{2}$. That is, $X_{\Delta} \subset \mathbb{P}^{n-1}=\mathbb{P}\left(\mathbb{C}^{n}\right)$ is the closure of the set

$$
X_{\Delta}^{\circ}=\left\{\left(x^{a_{1}}: \cdots: x^{a_{n}}\right): x=\left(x_{1}, x_{2}\right) \in\left(\mathbb{C}^{*}\right)^{2}\right\},
$$

where $\Delta \cap \mathbb{Z}^{2}=\left\{a_{1}, \ldots, a_{n}\right\}$. We can identify the projectivization of the dual space of $\mathbb{C}^{n}, \mathbb{P}\left(\left(\mathbb{C}^{n}\right)^{*}\right)$, as the projectivization of the vector space of Laurent polynomials whose Newton polygons are subsets of the polygon $\Delta$, which is called the tautological linear system of curves on the toric surface $X_{\Delta}$ and denoted by $\mathbb{P}_{\Delta}$. We study 
several subvarieties $\mathbb{V}$ • of this linear system $\mathbb{P}_{\Delta}$. The study of $\mathbb{V}$ • was motivated by trying to understand the initial schemes of Severi varieties. However, besides the roles as building blocks to understand the Severi varieties, the author believes that the $\mathbb{V}_{\bullet}$ s have their own independent interests and also they may be generalized in many different perspectives. Let $\mathbb{T}_{\Delta}$ be the big open torus of $\mathbb{P}_{\Delta}$.

\section{1. $\mathbb{V}_{\partial \mathcal{S}(\Delta), \mathbb{P}^{1}}$.}

Definitions 3.1. Let $\mathbb{V}_{\partial \Delta}$ denote the set of all $f \in \mathbb{T}_{\Delta}$ such that the restriction of $f$ on each edge of $\Delta$ is a pure power of a binomial (up to multiplication by a monomial), i.e. of the form of $x^{a} y^{b}\left(\alpha x^{c}+\beta y^{d}\right)^{s}$, where $a, b, c, d \in \mathbb{Z}, \alpha, \beta \in \mathbb{C}^{*}$ and $s$ is the lattice length of the edge. Geometrically, points of $\mathbb{V}_{\partial \Delta}$ correspond to curves on the toric surface $X_{\Delta}$ such that they cross the union of the toric divisors at precisely $l$ points, where $l$ is the number of edges of $\Delta$. More generally, we consider subdivisions $\mathcal{S}(\Delta)$ of the polygon $\Delta$. Let $\mathbb{V}_{\partial \mathcal{S}(\Delta)}$ denote the set of all $f \in \mathbb{T}_{\Delta}$ such that $f_{\Delta_{i}} \in \mathbb{V}_{\partial \Delta_{i}}$ for every $\Delta_{i} \in \operatorname{Faces}(\mathcal{S}(\Delta))$, where $f_{\Delta_{i}}$ is the restriction of $f$ on $\Delta_{i}$. Let $\mathbb{V}_{\partial \mathcal{S}(\Delta), \mathbb{P}^{1}}$ denote the set of all $f \in \mathbb{V}_{\partial \mathcal{S}(\Delta)}$ such that $f_{\Delta_{i}}$ defines a rational curve which is unibranch at each intersection point with the boundary divisors of the toric surface $X_{\Delta_{i}}$ for every $\Delta_{i} \in \operatorname{Faces}(\mathcal{S}(\Delta))$.

Lemma 3.2. [22, Lemma 3.5.] If $\Delta=\boldsymbol{\Delta}$ is a triangle, every $f \in \mathbb{V}_{\partial \mathbf{\Delta}, \mathbb{P}^{1}}$ can be given by the following rational parametrization,

$$
\theta \mapsto\left(\alpha \theta^{s_{1} v_{11}}(\theta+1)^{s_{2} v_{21}}, \beta \theta^{s_{1} v_{12}}(\theta+1)^{s_{2} v_{22}}\right),
$$

where $\alpha, \beta \in\left(\mathbb{C}^{*}\right)^{2}$ and $v_{1}=\left(v_{11}, v_{12}\right), v_{2}=\left(v_{21}, v_{22}\right)$ are two vectors among the three primitive inner-normal vectors to the edges of the triangle $\boldsymbol{\Lambda}$, and $s_{1}$ and $s_{2}$ are the lattice lengths of the corresponding edges of $\mathbf{\Delta}$.

Theorem 3.3. Suppose that $\mathcal{S}(\Delta)$ is triangular, that is, every face $\Delta_{i}$ of $\mathcal{S}(\Delta)$ is a triangle. Then the following hold true.

(1) $\mathbb{V}_{\partial \mathcal{S}(\Delta), \mathbb{P}^{1}}$ is a translation of a closed subgroup of the torus $\mathbb{T}_{\Delta}$.

(2) Its dimension is equal to $|\operatorname{Vertices}(\mathcal{S}(\Delta))|-1$.

Proof. There are three steps to complete the proof. First, we show that $\mathbb{V}:=$ $\mathbb{V}_{\partial \mathcal{S}(\Delta), \mathbb{P}^{1}}$ is not empty. Second, we construct a closed subgroup $\mathbb{G}$ of $\mathbb{T}_{\Delta}$ with dimension $|\operatorname{Vertices}(\mathcal{S}(\Delta))|-1$. Last, we show that $\mathbb{V}$ is equal to the translation $f \cdot \mathbb{G}$ of any point $f \in \mathbb{V}$.

Step 1. From the Lemma 3.2 , we know that for a triangle $\Delta$ any element in $\mathbb{V}_{\partial \Delta, \mathbb{P}^{1}}$ is uniquely determined by an element $(\alpha, \beta) \in\left(\mathbb{C}^{*}\right)^{2}$ by assuming that one of the vertices of $\Delta$ is the origin and the constant term of an equation is always 1 . Let us denote this element by $f^{(\alpha, \beta)}$. We extend this argument to the many-triangles case, $\mathcal{S}(\Delta): \Delta_{1} \cup \cdots \cup \Delta_{m}$. We know that we can always find a $(\alpha, \beta) \in\left(\mathbb{C}^{*}\right)^{2}$ such that $f^{(\alpha, \beta)}$ (up to the multiplication by a monomial) satisfies a given prescription on any two of the three edges of a triangle $\Delta$. That is, the following data are prescribed: the coefficients of the equation $f^{(\alpha, \beta)}$ at the vertices of $\Delta$ and the intersection points of the rational curve (defined by $f^{(\alpha, \beta)}$ ) with toric divisors corresponding to two of the three edges of $\Delta$. (for a proof, see [22, Lemma 3.5.]) Now we choose an orientation on the adjacency graph $\mathcal{S}(\Delta)^{*}$ of $\mathcal{S}(\Delta)$ which has no oriented cycle and no sink at vertices of 3 -valency. (see $\$ 2.3 .1$ for details.) It is clear that such oriented adjacency graph provides an algorithm to construct a point in $\mathbb{V}$. That is, we can 
choose a consistent collection of $(\alpha, \beta)_{\Delta_{i}}$ for sub-polygons $\Delta_{i}$ in $\mathcal{S}(\Delta)$. Therefore $\mathbb{V}$ is not empty.

Step 2. Choose a linear order, $\Delta_{1}, \ldots, \Delta_{m}$, in the set of triangles in the subdivision $\mathcal{S}(\Delta)$. We choose one of the vertices of $\Delta_{1}$ and assume that it is the origin. We order the set of inner edges in $\mathcal{S}(\Delta)$ in the following way: Choose all inner edges belonging to $\Delta_{1}$ (there are at most three such edges). Put them in an order. Then, choose all inner edges belonging to $\Delta_{2}$ except the ones which may belong to $\Delta_{1}$. Add them in an order to the first set. In this way, we put a linear order in the set of all inner edges. Each inner edge defines two binomial equations as follows: Let $l=s_{i j}$ be the inner edge shared by $\Delta_{i}$ and $\Delta_{j}, i<j$. Let $a=\left(a_{1}, a_{2}\right)$ be the lattice point of one of the two ends of $l$ and let $v=\left(v_{1}, v_{2}\right)$ be the primitive vector along $l$ from $a$.

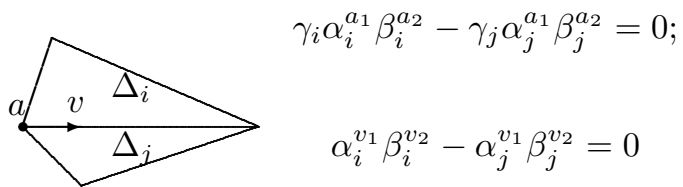

We collect the binomials for all inner edges and add one more binomial, $\gamma_{1}=1$. Let us denote this system by $(\star)$. Then it is clear that this system is uniquely determined by the following system,

$$
\begin{gathered}
\gamma_{1}=1 ; \\
\alpha_{i}^{v_{1}} \beta_{i}^{v_{2}} \alpha_{j}^{-v_{1}} \beta_{j}^{-v_{2}}=1(\star \star)
\end{gathered}
$$

where the monomials in the left hand side of the equations are collected for all the inner edges. Let $\mathbb{G}$ be the closed subgroup in the torus $\left(\mathbb{C}^{*}\right)^{3} \times \cdots \times\left(\mathbb{C}^{*}\right)^{3}$ with

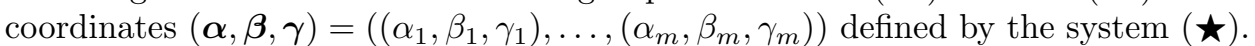
Let $M:=M_{\partial \mathcal{S}}(\Delta), \mathbb{P}^{1}$ be the matrix corresponding to the monomials in the left hand side of equations in $(\star \star)$ where the rows are indexed by the inner edges and the columns are indexed by $\left(\alpha_{i}, \beta_{i}\right), i=1, \ldots, m$. It is straightforward to see that the rows of $M$ are linearly independent. Therefore,

$$
\operatorname{dim}(\mathbb{G})=2|\operatorname{Triangles}(\mathcal{S}(\Delta))|-|\operatorname{IEdges}(\mathcal{S}(\Delta))|,
$$

where IEdges $(\mathcal{S}(\Delta))$ is the set of all inner edges in $\mathcal{S}(\Delta)$. Also, by the Lemma 3.5 .

$$
\operatorname{dim}(\mathbb{G})=|\operatorname{Vertices}(\mathcal{S}(\Delta))|-1 .
$$

Now we embed $\mathbb{G}$ into $\mathbb{T}_{\Delta}$ in the following way,

$$
\begin{gathered}
\Phi=\left(\Phi_{\left(w_{1}, w_{2}\right)}\right)_{\left(w_{1}, w_{2}\right) \in \Delta \cap \mathbb{Z}^{2}}: \mathbb{G} \rightarrow \mathbb{T}_{\Delta}, \\
\Phi_{\left(w_{1}, w_{2}\right)}\left(\left(\alpha_{1}, \beta_{1}, \gamma_{1}\right), \ldots,\left(\alpha_{m}, \beta_{m}, \gamma_{m}\right)\right)=\gamma_{k} \alpha_{k}^{w_{1}} \beta_{k}^{w_{2}},
\end{gathered}
$$

where $\left(w_{1}, w_{2}\right) \in \Delta_{k} \cap \mathbb{Z}^{2}, k=1, \ldots, m$. This map is well-defined because $\mathbb{G}$ satisfies the system $(\star)$. Also this map is injective by the Lemma 3.6 .

Step 3. Let us first show that $f \cdot \mathbb{G} \subset \mathbb{V}$ for any $f \in \mathbb{V}$. (We don't distinguish $\mathbb{G}$ from its image under the embedding $\Phi$.) Let $(\boldsymbol{\alpha}, \boldsymbol{\beta}, \boldsymbol{\gamma}) \in \mathbb{G}$. The restriction of $f \cdot(\boldsymbol{\alpha}, \boldsymbol{\beta}, \boldsymbol{\gamma})$ on $\Delta_{k}$ is given by $\gamma_{k} f_{\Delta_{k}}\left(\alpha_{k} x, \beta_{k} y\right)$, which is a point in $\mathbb{V}_{\Delta_{k}, \mathbb{P}^{1}}(k=$ $1, \ldots, m)$. Thus, it is enough to show that the restrictions of $f \cdot(\boldsymbol{\alpha}, \boldsymbol{\beta}, \boldsymbol{\gamma})$ on all subtriangles coincide along the inner edges. It follows from the fact that $\mathbb{G}$ satisfies the $\operatorname{system}(\star)$. Now we show that the other inclusion also holds. Let $h, h^{\prime} \in \mathbb{V}$. Then 
JIHYEON JESSIE YANG

the restriction of $h$ (resp. $h^{\prime}$ ) on $\Delta_{k}$ has the following form up to the multiplication by a monomial $x^{b_{1}} y^{b_{2}}$,

$$
\begin{aligned}
& h_{\Delta_{k}}(x, y)=\gamma_{k}\left(f^{\left(\alpha_{k}, \beta_{k}\right)}\right)=\gamma_{k} f^{\left(\alpha_{k}, \beta_{k}\right)}\left(\alpha_{k} x, \beta_{k} y\right) \\
& h_{\Delta_{k}}^{\prime}(x, y)=\gamma_{k}^{\prime}\left(f^{\left(\alpha_{k}^{\prime}, \beta_{k}^{\prime}\right)}\right)=\gamma_{k}^{\prime} f^{\left(\alpha_{k}^{\prime}, \beta_{k}^{\prime}\right)}\left(\alpha_{k}^{\prime} x, \beta_{k}^{\prime} y\right),
\end{aligned}
$$

for some $\left(\alpha_{k}, \beta_{k}, \gamma_{k}\right)$ (resp. $\left.\left(\alpha_{k}^{\prime}, \beta_{k}^{\prime}, \gamma_{k}^{\prime}\right) \in\left(\mathbb{C}^{*}\right)^{3}\right),\left(b_{1}, b_{2}\right) \in \Delta \cap \mathbb{Z}^{2}$, where $k=$ $1, \ldots, m$. Thus $h_{\Delta_{k}}^{\prime}(x, y)=\gamma_{k}^{\prime} \gamma_{k}^{-1} h_{\Delta_{k}}\left(\alpha_{k}^{-1} \alpha_{k}^{\prime} x, \beta_{k}^{-1} \beta_{k}^{\prime} y\right)$. That is, $h_{\Delta_{k}}^{\prime}$ is the restriction of $\left(\boldsymbol{\alpha}^{-1} \boldsymbol{\alpha}^{\prime}, \boldsymbol{\beta}^{-1} \boldsymbol{\beta}^{\prime}, \boldsymbol{\gamma}^{\prime} \boldsymbol{\gamma}^{-1}\right) \cdot h$ on $\Delta_{k}$. Therefore

$$
h^{\prime}=\left(\boldsymbol{\alpha}^{-1} \boldsymbol{\alpha}^{\prime}, \boldsymbol{\beta}^{-1} \boldsymbol{\beta}^{\prime}, \boldsymbol{\gamma}^{\prime} \boldsymbol{\gamma}^{-1}\right) \cdot h .
$$

We have completed the proof.

Remark 3.4. We can compute the number of components of $\mathbb{V}_{\partial \mathcal{S}(\Delta), \mathbb{P}^{1}}$ easily from

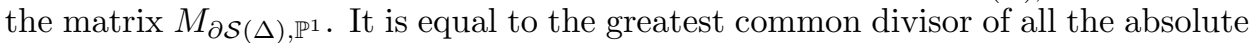
values of $l \times l$ minors of $M_{\partial \mathcal{S}}(\Delta), \mathbb{P}^{1}$. Also it is equal to the number of lattice points in the parallelepiped $P=\left\{x_{1} v_{1}+\cdots+x_{l} v_{l}: 0 \leq x_{i}<1, i=1, \ldots, l\right\}$, where $v_{1}, \ldots, v_{l}$ are the row vectors of $M_{\partial \mathcal{S}}(\Delta), \mathbb{P}^{1}$.

Lemma 3.5. If a subdivision $\mathcal{S}(\Delta)$ of $\Delta$ is triangular, then

$$
2|\operatorname{Triangles}(\mathcal{S}(\Delta))|-|\operatorname{IEdges}(\mathcal{S}(\Delta))|=|\operatorname{Vertices}(\mathcal{S}(\Delta))|-1
$$

Proof. Let $F$ be the number of the triangles, $E^{\prime}$ be the number of the edges on the boundary of $\Delta$, and let $I E$ be the number of the inner edges in $\mathcal{S}(\Delta)$, respectively. Then, $3 \cdot F=E^{\prime}+2 \cdot I E$. Since $\mid$ Vertices $|-|$ Edges $|+|$ Faces $\mid=1$,

$$
\begin{aligned}
|\operatorname{Vertices}(\mathcal{S}(\Delta))|-1 & =\mid \text { Edges }|-| \text { Faces } \mid \\
& =\left(E^{\prime}+I E\right)-F \\
& =(3 \cdot F-I E)-F \\
& =2 \cdot F-I E .
\end{aligned}
$$

Lemma 3.6. The map $\Phi=\left(\Phi_{\left(w_{1}, w_{2}\right)}\right)_{\left(w_{1}, w_{2}\right) \in \Delta \cap \mathbb{Z}^{2}}$ defined in the proof of Theorem 3.3 is injective.

Proof. Given a vertex $v_{1}$ of any triangle $\Delta_{k},(k=1, \ldots, m)$, we can find two lattice points $v_{2}, v_{3}$ on $\Delta_{k}$ such that the convex hull of $v_{1}, v_{2}, v_{3}$ is a primitive triangle, that is, it has no interior lattice point. Now it is clear that any given values for $\Phi_{v_{i}}(i=1,2,3)$ uniquely determine $\alpha_{k}, \beta_{k}, \gamma_{k}$.

Example 3.7. Let $\mathcal{S}(\Delta)$ be the following subdivision with 32 -dimensional faces $F_{1}, F_{2}, F_{3}$. Since $\mathcal{S}(\Delta)$ has no interior lattice point, $\mathbb{V}_{\partial \mathcal{S}(\Delta)}=\mathbb{V}_{\partial \mathcal{S}(\Delta), \mathbb{P}^{1}}$. We get

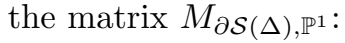

$$
M_{\partial \Delta, \mathbb{P}^{1}}=\left(\begin{array}{ccccccc} 
& \alpha_{1} & \beta_{1} & \alpha_{2} & \beta_{2} & \alpha_{3} & \beta_{3} \\
s_{12} & 1 & 2 & -1 & -2 & 0 & 0 \\
s_{13} & 1 & 0 & 0 & 0 & -1 & 0 \\
s_{23} & 0 & 0 & -1 & 2 & 1 & -2
\end{array}\right)
$$

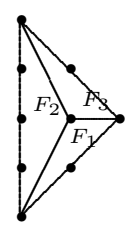

The Smith Normal Form of $M_{\partial \mathcal{S}(\Delta), \mathbb{P}^{1}}$ is :

$$
\left(\begin{array}{llllll}
1 & 0 & 0 & 0 & 0 & 0 \\
0 & 1 & 0 & 0 & 0 & 0 \\
0 & 0 & 2 & 0 & 0 & 0
\end{array}\right)
$$


Thus $\mathbb{V}_{\partial \mathcal{S}(\Delta)}=\mathbb{V}_{\partial \mathcal{S}(\Delta), \mathbb{P}^{1}}$ is a union of two translations of 3-dimensional subtorus of $\mathbb{T}_{\Delta}$.

3.2. $\mathbb{V}_{\partial \mathcal{S}(\Delta), \text { nodal }}$. Now we allow to have parallelograms in a subdivision $\mathcal{S}(\Delta)$.

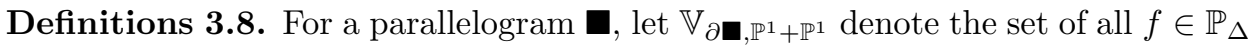
such that $f$ is the product of Laurent polynomials $f_{1}, f_{2}$ whose Newton polygons are two nonparallel sides of $\mathbf{a}$ and each $f_{i}$ is a pure power of a binomial for $i=1,2$. Let $\mathcal{A}^{c} \subset \boldsymbol{\square} \cap \mathbb{Z}^{2}$ be the set of lattice points in which are not in the lattice generated by the primitive vectors along the sides of $\boldsymbol{\square}$. Any element of $\mathcal{A}^{c}$ is called special. If $\mathcal{A}^{c}$ is not empty, then $\mathbf{\square}$ is called non-primitive.

(The figure on the right shows that there are 5 lattice points in

The unique interior lattice point is special.)

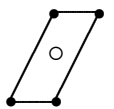

Notice that $f$ has no term corresponding to the monomial $x^{a}$ for any $f \in \mathbb{V}_{\partial \mathbf{m}, \mathbb{P}^{1}+\mathbb{P}^{1}}$ and $a \in \mathcal{A}^{c}$. Therefore $\mathbb{V}_{\partial} \boldsymbol{\mathbb { P } ^ { 1 } + \mathbb { P } ^ { 1 }}$ is contained in the coordinate subspace of $\mathbb{P}$ defined by the linear equations $z_{a}=0, a \in \mathcal{A}^{c}$. If $\mathcal{A}^{c}$ is empty, then the coordinate space is the ambient space $\mathbb{P}_{\mathbf{m}}$ itself. Let $\mathbb{T}_{\mathcal{A}}$ be the big open torus of this coordinate subspace.

Now suppose that $\mathcal{S}(\Delta):=\Delta_{1} \cup \cdots \cup \Delta_{m}$ is nodal, that is, every sub-polygon is either a triangle or a parallelogram. Let $\mathbb{V}_{\partial \mathcal{S}(\Delta) \text {,nodal }}$ denote the set of all $f \in \mathbb{P}_{\Delta}$ with the following properties,

- (ム) For every triangle $\Delta_{i}, f_{\Delta_{i}} \in \mathbb{V}_{\partial \Delta_{i}, \mathbb{P}^{1}}$;

- (ם) For every parallelogram $\Delta_{j}, f_{\Delta_{j}} \in \mathbb{V}_{\partial \Delta_{j}, \mathbb{P}^{1}+\mathbb{P}^{1}}$

We consider the subset $\mathcal{A}=\mathcal{A}_{1} \cup \cdots \cup \mathcal{A}_{m}$ of $\Delta \cap \mathbb{Z}^{2}$ defined as follows:

- if $\Delta_{i}$ is a triangle, $\mathcal{A}_{i}=\Delta_{i} \cap \mathbb{Z}^{2}$;

- if $\Delta_{i}$ is a parallelogram, $\mathcal{A}_{i}$ is the set of non-special lattice points on $\Delta_{i}$.

\section{Theorem 3.9.}

(1) $\mathbb{V}_{\partial \mathcal{S}(\Delta), \text { nodal }}$ is a translation of a closed subgroup of the torus $\mathbb{T}_{\mathcal{A}}$;

(2) Its dimension is equal to $|\operatorname{Vertices}(\mathcal{S}(\Delta))|-1-|\operatorname{Parallelograms}(\mathcal{S}(\Delta))|$.

Proof. The proof of the first statement follows from a simple adjustment of the proof of Theorem 3.3. Now the adjacency graph $\mathcal{S}(\Delta)^{*}$ may have vertices of 4 valency. We choose a directed graph on $\mathcal{S}(\Delta)^{*}$ which has no oriented cycle and no sink at vertices of 3 and 4 -valency and also the edges in $\mathcal{S}(\Delta)^{*}$ which are dual to the parallel edges of a parallelogram in $\mathcal{S}(\Delta)$ are co-oriented. Using this directed

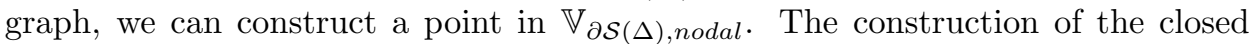
subgroup $\mathbb{G}$ is exactly same as the one given in the proof of Theorem 3.3 .

The second statement follows from the following Lemma 3.10 .

Lemma 3.10. For a nodal subdivision $\mathcal{S}(\Delta)$, the following holds true:

(3.18) $2 \mid$ Triangles $|+2|$ Parallelograms $|-|$ IEdges $|=|$ Vertices $|-1-|$ Parallelograms $\mid$

Proof. Let $T:=\mid$ Triangles $|, P:=|$ Parallelograms $|, E:=|$ Edges $|, I E:=|$ IEdges $\mid, V:=$ $\mid$ Vertices $\mid$, and $F:=\mid$ Faces $\mid$. Then $V-E+F=1, F=T+P$, and $3 T+4 P=E+I E$. Thus $V-1-P=E-T-2 P=(3 T+4 P-I E)-T-2 P=2 T+2 P-I E$.

Remark 3.11. In terms of the algebra of tropical cycles $\mathbf{A}(22.4)$, we can write:

$$
\operatorname{Trop}\left(\mathbb{V}_{\partial \mathcal{S}(\Delta), \text { nodal }}\right)=l(\mathbb{V}) \cdot \operatorname{Trop}\left(\mathbb{G}^{e}\right),
$$

where $l(\mathbb{V})$ is the number of components of $\mathbb{V}=\mathbb{V}_{\partial \mathcal{S}(\Delta), \text { nodal }}$, and $\mathbb{G}^{e}$ is the identity component of the closed subgroup $\mathbb{G}=\mathbb{G}_{\partial \mathcal{S}(\Delta), \text { nodal }}$ of $\mathbb{T}_{\mathcal{A}}$. 


\section{Tropical Severi Varieties}

4.1. Severi varieties. Severi varieties are very classical varieties which go back to F. Enriques [6] and F.Severi [20. As in $\$ 3$ let $X_{\Delta}$ be the projective toric surface constructed from a 2-dimensional convex lattice polygon $\Delta$, let $\mathbb{P}_{\Delta}$ be the tautological linear system on $X_{\Delta}$ and let $\mathbb{T}_{\Delta}$ be the big open torus of $\mathbb{P}_{\Delta}$.

Definition 4.1. Let $\delta$ be a non-negative integer with $\delta \leq\left|\operatorname{Int}(\Delta) \cap \mathbb{Z}^{2}\right|$.

- The Severi variety $\operatorname{Sev}(\Delta, \delta) \subset \mathbb{P}_{\Delta}$ is the closure of the set of curves with exactly $\delta$ nodes (ordinary double points) as their only singularities.

- The very affine Severi variety $\operatorname{Sev}(\Delta, \delta)^{\circ}$ is the intersection of the Severi variety $\operatorname{Sev}(\Delta, \delta)$ with the big open torus $\mathbb{T}_{\Delta}$.

- The tropicalization of the very affine Severi variety is called tropical Severi variety and denoted by $\operatorname{Trop}(\operatorname{Sev}(\Delta, \delta))$.

It is well known that $\operatorname{dim}(\operatorname{Sev}(\Delta, \delta))$ is equal to $\left|\Delta \cap \mathbb{Z}^{2}\right|-\delta-1$. We only consider the case when $\operatorname{Sev}(\Delta, \delta)^{\circ}$ is dense in $\operatorname{Sev}(\Delta, \delta)$ and thus they have the same dimension. This case includes the classical one, plane curves of a given degree $d$, equivalently, the case when the polygon $\Delta$ is the triangle with vertices $(0,0),(d, 0),(0, d)$, where $d$ is a positive integer.

4.2. Patchworking Theory. In this section we review Shustin's patchworking theory. In 1979-80, O. Viro found a patchworking construction for obtaining real nonsingular projective algebraic hypersurfaces with prescribed topology. This method was a breakthrough in Hilbert's 16th problem. In the early 1990's, E. Shustin suggested to use the patchworking construction for tracing other properties of objects defined by polynomials, for example, prescribed singularities of algebraic hypersurfaces and many others 21, 22. He starts with a modified version of the patchworking construction, which allows one to keep singularities in the patchworking deformation. An important difference with respect to the original Viro method is that singularities are not stable in general, and thus one has to modify the Viro deformation and impose certain transversality conditions.

The following is a version of Shustin's patchworking theory about curves on toric surfaces, summarized for the purposes of this paper [22, §3.7]. (Note the conventions in $\sqrt{2.2}$ )

- Let $\mathcal{S}(\Delta)$ be a regular subdivision of $\Delta$ with $\operatorname{rank}(\mathcal{S}(\Delta))=\operatorname{dim}(\operatorname{Sev}(\Delta, \delta))$. Suppose that $\mathcal{S}(\Delta)$ is simple and nodal, then there exists a c-vector $\omega$ of $\operatorname{Sev}(\Delta, \delta)$ such that $\Delta_{\omega}=\mathcal{S}(\Delta)$.

- Let $\omega: \Delta \cap \mathbb{Z}^{2} \rightarrow \mathbb{Z}$ be an integral-valued function on $\Delta \cap \mathbb{Z}^{2}$ such that $\operatorname{rank}(\omega)=\operatorname{dim}(\operatorname{Sev}(\Delta, \delta))$ and $\Delta_{\omega}$ is simple-triangular.

(Enumeration 1) If we fix the coefficients $c_{b} \in \mathbb{C} \backslash\{0\}$ for $b \in \operatorname{Vertices}\left(\Delta_{\omega}\right)$, then the number of $F \in \mathbb{V}=\mathbb{V}_{\partial \Delta_{\omega}, \text { nodal }}$ with $F(b)=c_{b}$ is equal to

$$
\frac{\prod 2 \text { area }(\text { Triangles })}{\prod \text { length }\left(\operatorname{Edges}\left(\Delta_{\omega}\right)\right)},
$$

where the numerator stands for the product of twice the (Euclidean) area of each triangle in $\Delta_{\omega}$ and the denominator is the product of the lattice lengths of the edges.

(Enumeration 2) If we fix the coefficients $c_{b}(t)=\bar{c}_{b} t^{\omega(b)}+$ l.o.t. $\in \mathbb{K} \backslash\{0\}$ for $b \in \operatorname{Vertices}\left(\Delta_{\omega}\right)$, then the number of $f \in \operatorname{Sev}(\Delta, \delta)(\mathbb{K})$ with $f(b)=$ 
$c_{b}(t)$ and $\tau_{f}$ dual to $\Delta_{\omega}$ is equal to

$$
\prod \text { 2area(Triangles). }
$$

(In Shustin's notations in [22, §3.7], given $c_{b}(t)$ for $b \in \operatorname{Vertices}\left(\Delta_{\omega}\right)$, the number of possible $A$ (amoeba) is 1 , the number of possible $F$ (initial terms of coefficients of $f$ ) is $\frac{\prod 2 \text { area(Triangles) }}{\prod \text { length }\left(\operatorname{Edges}\left(\Delta_{\omega}\right)\right)}$, and the number of possible $R$ (deformation patterns) is $\prod$ length $\left(\operatorname{Edges}\left(\Delta_{\omega}\right)\right)$. Thus the number of possible $(A, F, R)$ is equal to $\prod 2$ area(Triangles) and each of them gives rise to a unique $f \in \operatorname{Sev}(\Delta, \delta)(\mathbb{K})$.)

Remark 4.2. In fact the enumerations above hold when the subdivision $\Delta_{\omega}$ is simple-nodal, which was the case Shustin worked on. In this case, we replace $\operatorname{Vertices}\left(\Delta_{\omega}\right)$ by a subset $\mathcal{B}$ with $|\mathcal{B}|=\operatorname{dim}(\operatorname{Sev}(\Delta, \delta))+1$ so that for any $F \in \mathbb{V}$ fixing coefficients for $b \in \mathcal{B}$ uniquely determines the other coefficients for $b \in$ $\operatorname{Vertices}\left(\Delta_{\omega}\right) \backslash \mathcal{B}$. Also the denominator of the formula 4.1 should be adjusted as follows: $\widetilde{\prod}$ length $\left(\operatorname{Edges}\left(\Delta_{\omega}\right)\right)$, the product of the lattice lengths of the edges which are representatives of each equivalence class in $\operatorname{Edges}\left(\Delta_{\omega}\right)$, where we define an equivalence relation as follows: let $e \sim e^{\prime}$ if $e$ and $e^{\prime}$ are the parallel edges of a parallelogram in $\Delta_{\omega}$ and extend it by transitivity.

4.3. Initial schemes of very affine Severi varieties. Let $\omega \in \mathbb{Z}^{\Delta \cap \mathbb{Z}^{2}}$ be an integral vector. As in $\$ 2.2 .1$, we have the initial scheme $\operatorname{in}_{\omega} \operatorname{Sev}(\Delta, \delta) \subset \mathbb{T}_{\Delta}$ of the very affine Severi variety $\operatorname{Sev}(\Delta, \delta)^{\circ}$. Also, $\omega$ can be identified with an integralvalued function on the set of lattice points $\Delta \cap \mathbb{Z}^{2}$ on $\Delta$ and we get the regular subdivision $\Delta_{\omega}$ of $\Delta$ constructed from $\omega$. We are going to find a description of $\operatorname{in}_{\omega} \operatorname{Sev}(\Delta, \delta)$ with respect to $\Delta_{\omega}$. Let us begin with an example which is simple since the Severi variety is a hypersurface:

Example 4.3. Let $\Delta$ be as described on the right. A general polynomial with Newton polygon $\Delta$ is written as $f=a y^{2}+b x^{2} y+c x y+d y+e$.

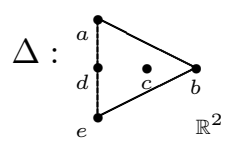
We consider the curves $V(f)$ with one singular point, that is, $\operatorname{Sev}(\Delta, 1)$.

The hypersurface $\operatorname{Sev}(\Delta, 1)$ is defined by a polynomial $D_{\mathcal{A}}$ which is called $\mathcal{A}$ discriminant, where $\mathcal{A}=\Delta \cap \mathbb{Z}^{2}$ :

$$
D_{\mathcal{A}}=16 b^{2} d^{2}-8 b c^{2} d+c^{4}-64 a b^{2} e
$$

The figure below on the left represents the Newton polytope of $D_{\mathcal{A}}$ and $\operatorname{Trop}(\operatorname{Sev}(\Delta, 1))$ which is modded out by the 3 -dimensional linearity space. The figure on the right shows the connection to the subdivisions of $\Delta$.
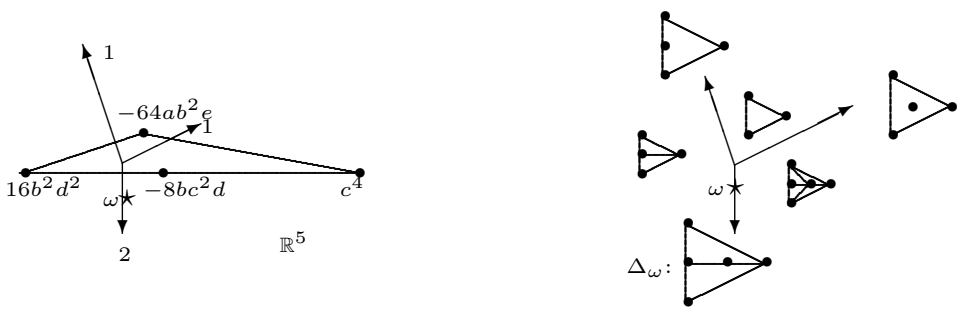

Choose an integral vector $\omega$ on one of the rays of $\operatorname{Trop}(\operatorname{Sev}(\Delta, 1))$ as shown on the figure above. The corresponding initial scheme $\operatorname{in}_{\omega} \operatorname{Sev}(\Delta, 1)$ is defined by the polynomial $16 b^{2} d^{2}-8 b c^{2} d+c^{4}=\left(4 b d-c^{2}\right)^{2}$. That is, $\operatorname{in}_{\omega} \operatorname{Sev}(\Delta, 1)$ is a non-reduced 
scheme, a translation of the torus $V\left(b d-c^{2}\right)$ with multiplicity 2 . Let us look at the corresponding subdivision $\Delta_{\omega}$. We can find that $l(\mathbb{V})$, the number of components of $\mathbb{V}=\mathbb{V}_{\partial \Delta_{\omega}, \text { nodal }}$, is equal to 1 . Also we see that $\Delta_{\omega}$ has one interior edge of lattice length 2. Therefore, the number of translations of a subtorus (counted with multiplicity) in the initial scheme $\operatorname{in}_{\omega} \operatorname{Sev}(\Delta, 1)$ coincides with the product of $l(\mathbb{V})$ and the lattice length of the interior edge of $\Delta_{\omega}$. The main theorems of this paper presented below show that this description of the initial schemes of Severi varieties holds true in general.

The following theorem gives a description of the support of $\operatorname{Trop}(\operatorname{Sev}(\Delta, \delta))$.

Theorem 4.4. If the rank of $\omega$ is strictly larger than $\operatorname{dim}(\operatorname{Sev}(\Delta, \delta))$, then $\omega$ is not a c-vector of $\operatorname{Sev}(\Delta, \delta)$, that is, $\operatorname{in}_{\omega} \operatorname{Sev}(\Delta, \delta)=\emptyset$.

Proof. Suppose $\omega$ is a c-vector of $\operatorname{Sev}(\Delta, \delta)$ with $\operatorname{rank}(\omega) \geq \operatorname{dim}(\operatorname{Sev}(\Delta, \delta))$. In 2.3 .2 we showed that $\omega$ must satisfy Shustin's combinatorial characterization, i.e., the subdivision $\Delta_{\omega}$ is simple-nodal and $\operatorname{rank}(\omega)=\operatorname{dim}(\operatorname{Sev}(\Delta, \delta))$. Thus the rank of any c-vector of $\operatorname{Sev}(\Delta, \delta)$ cannot by strictly larger than $\operatorname{dim}(\operatorname{Sev}(\Delta, \delta))$.

Also if $\omega$ is a c-vector of $\operatorname{Sev}(\Delta, \delta)$ with maximal rank $\operatorname{dim}(\operatorname{Sev}(\Delta, \delta))$, Shustin's combinatorial characterization says that the regular subdivision $\Delta_{\omega}$ must be simple and nodal. Furthermore, if we impose one more restriction on the parallelograms in $\Delta_{\omega}$, we can obtain a complete description of initial schemes $\operatorname{in}_{\omega} \operatorname{Sev}(\Delta, \delta)$ as presented in the next theorem following lemma which provides a geometric characterization of initial schemes.

Lemma 4.5. Let $X$ be a subvariety of an algebraic torus $\mathbb{T}$. The set of closed points of $\operatorname{in}_{\omega} X$ is equal to

$$
\left\{\bar{z} \in \mathbb{T}: \text { there exists } z=\bar{z} t^{\omega}+\text { l.o.t. } \in X(\mathbb{K}) \subset \mathbb{T}(\mathbb{K})\right\},
$$

where l.o.t. stands for "lower order terms" and $z$ is in vector-notation.

Proof. The proof for the inclusion $\subset$ can be found in [12, Lemma 4.15], [13, Proposition 4], [19. Let us consider the other inclusion $\supset$. Suppose $z=\bar{z} t^{\omega}+$ l.o.t. $\in X(\mathbb{K})$ and let $f \in I(X)$. It is enough to show that $\operatorname{in}_{\omega} f(\bar{z})=0$, which follows from the fact that $\operatorname{in}_{\omega} f(\bar{z})$ is the constant term of $f(z) \cdot t^{-\gamma} \in \mathbb{C}[t]$, where $\gamma$ is the $t-\omega$-degree of $f$.

Theorem 4.6. Let $\omega$ be a c-vector of $\operatorname{Sev}(\Delta, \delta)$. Suppose that $\omega$ satisfies the following conditions:

- The rank of $\omega$ is maximal, that is, $\operatorname{rank}(\omega)=\operatorname{dim}(\operatorname{Sev}(\Delta, \delta))$;

- The regular subdivision $\Delta_{\omega}$ has no non-primitive parallelogram.

Then the following hold true:

(1) As the sets of closed points, $\operatorname{in}_{\omega} \operatorname{Sev}(\Delta, \delta)$ is equal to $\mathbb{V}=\mathbb{V}_{\partial \Delta_{\omega}, \text { nodal }}$. Thus, $\operatorname{in}_{\omega} \operatorname{Sev}(\Delta, \delta)$ is a union of finitely many translations of the torus $\mathbb{G}^{e}$, the identity component of $\mathbb{G}=\mathbb{G}_{\partial \Delta_{\omega}, \text { nodal }}$.

(2) The weight of $\omega$ on $\operatorname{in}_{\omega} \operatorname{Sev}(\Delta, \delta)$, that is, the number of such translations of the torus $\mathbb{G}^{e}$ counted with multiplicity, is equal to

$$
\boldsymbol{m}_{\mathrm{Sev}(\Delta, \delta)}(\omega)=l(\mathbb{V}) \cdot \widetilde{\prod} \operatorname{length}\left(\operatorname{Edges}\left(\Delta_{\omega}\right)\right)
$$

where

(a) $l(\mathbb{V})$ is the number of connected components of $\mathbb{V}$; 
(b) $\widetilde{\prod l}$ length $\left(\operatorname{Edges}\left(\Delta_{\omega}\right)\right)$ is the product of the lattice lengths of the edges which are representatives of each equivalence class in $\operatorname{Edges}\left(\Delta_{\omega}\right)$, where we define an equivalence relation as follows: let $e \sim e^{\prime}$ if $e$ and $e^{\prime}$ are the parallel edges of a parallelogram in $\Delta_{\omega}$ and extend it by transitivity.

Proof. We prove the first statement. Applying Lemma 4.5 to our case $X=$ $\operatorname{Sev}(\Delta, \delta)$, we see that $\bar{c}=\left(\bar{c}_{a}\right)_{a \in \Delta \cap \mathbb{Z}^{2}} \in \operatorname{in}_{\omega} \operatorname{Sev}(\Delta, \delta)$ if and only if there exists a 1-parameter equisingular family of nodal curves with $\delta$ nodes defined by

$$
f_{(t)}(x, y)=\sum_{a=\left(a_{1}, a_{2}\right) \in \Delta \cap \mathbb{Z}^{2}} c_{a}(t) x^{a_{1}} y^{a_{2}}
$$

such that

$$
c_{a}(t)=\bar{c}_{a} t^{\omega_{a}}+\text { l.o.t. }
$$

Thus, $\bar{c}$ is closely related to the tropicalization of $f_{(t)}$. Let us recall the definition of the tropicalization of $f_{(t)}$ : Let $\nu_{f_{(t)}}$ be the concave hull of $\omega=V a l_{f_{(t)}}$ and rewrite $f_{(t)}$ with respect to $\nu_{f_{(t)}}$ :

$$
f_{(t)}(x, y)=\sum_{a=\left(a_{1}, a_{2}\right) \in \Delta \cap \mathbb{Z}^{2}} c_{a}(t) x^{a_{1}} y^{a_{2}}
$$

such that

$$
c_{a}(t)=c_{a}^{\circ} t^{\nu_{f(t)}(a)}+\text { l.o.t. }
$$

where $c_{a}^{\circ}$ is a complex number such that $c_{a}^{\circ}=\bar{c}_{a}$ exactly when $\nu_{f_{(t)}}(a)=\omega(a)$ and otherwise $c_{a}^{\circ}=0$. This collection of complex numbers, $c^{\circ}=\left\{c_{a}^{\circ}: a \in \Delta \cap \mathbb{Z}^{2}\right\}$, together with the regular subdivision $\Delta_{\nu_{f(t)}}=\Delta_{\omega}: \Delta_{1} \cup \cdots \Delta_{m}$ of the Newton polygon $\Delta$ of $f_{(t)}$ gives rise to a collection of complex polynomials $f_{1}, \ldots, f_{m}$ with $\operatorname{Newton}\left(f_{i}\right)=\Delta_{i}$ for $i=1, \ldots, m$. Now Shustin's geometric characterization 2.14 implies that under the hypothesis of our theorem, if $\bar{c} \in \operatorname{in}_{\omega} \operatorname{Sev}(\Delta, \delta)$, then $\bar{c}=c^{\circ} \in$ $\mathbb{V}_{\partial \Delta_{\omega}, \text { nodal }}$. (Notice that in our case that there is no non-primitive parallelogram in the subdivision $\Delta_{\omega}, c^{\circ}(a) \neq 0$ for all $a \in \Delta \cap \mathbb{Z}^{2}$, which also implies that $\nu_{f_{(t)}}=\omega$, that is, $\omega$ should be concave.) Thus, $\operatorname{in}_{\omega} \operatorname{Sev}(\Delta, \delta)$ is a subset of $\mathbb{V}_{\partial \Delta_{\omega}, \text { nodal }}$. The other inclusion follows easily from Shustin's patchworking theory 4.2 .

Now we prove the second statement. Let $\mathcal{B}$ be a subset of $\operatorname{Vertices}\left(\Delta_{\omega}\right)$ with the properties given in Remark 4.2 and let $\mathbb{L}_{\mathcal{B}}$ be the $n-(\operatorname{dim}(\operatorname{Sev}(\Delta, \delta))+1)$ dimensional coordinate subspace of $\mathbb{T}_{\Delta}$ defined by the equations $x_{b}=1, \quad b \in \mathcal{B}$. Then Shustin's first enumeration (4.1) deduces the following:

$$
\left(\operatorname{Trop}(\mathbb{V}) \cdot \operatorname{Trop}\left(\mathbb{L}_{\mathcal{B}}\right)\right)=\frac{\prod 2 \operatorname{area}(\text { Triangles })}{\widetilde{\prod} \text { length }\left(\operatorname{Edges}\left(\Delta_{\omega}\right)\right)} .
$$

Moreover, from the second enumeration 4.2 , we obtain the following:

$$
\left(\left(\operatorname{Trop}\left(\operatorname{in}_{\omega} \operatorname{Sev}(\Delta, \delta)\right) \cdot \operatorname{Trop}\left(\mathbb{L}_{\mathcal{B}}\right)\right)\right)=\prod 2 \operatorname{area}(\text { Triangles }) .
$$


Thus,

(4.12)

$$
\begin{aligned}
\boldsymbol{m}_{\operatorname{Sev}(\Delta, \delta)}(\omega)\left(\operatorname{Trop}\left(\mathbb{G}^{e}\right) \cdot \operatorname{Trop}\left(\mathbb{L}_{\mathcal{B}}\right)\right) & =\left(\operatorname{Trop}\left(\operatorname{in}_{\omega} \operatorname{Sev}(\Delta, \delta)\right) \cdot \operatorname{Trop}\left(\mathbb{L}_{\mathcal{B}}\right)\right) \\
& =\prod 2 \operatorname{area}(\operatorname{Triangles}) \\
& =\widetilde{\prod} \operatorname{length}\left(\operatorname{Edges}\left(\Delta_{\omega}\right)\right) \cdot \frac{\prod \text { 2area }(\operatorname{Triangles})}{\widetilde{\Pi} \text { length }\left(\operatorname{Edges}\left(\Delta_{\omega}\right)\right)} \\
& =\widetilde{\prod} \operatorname{length}\left(\operatorname{Edges}\left(\Delta_{\omega}\right)\right)\left(\operatorname{Trop}(\mathbb{V}) \cdot \operatorname{Trop}\left(\mathbb{L}_{\mathcal{B}}\right)\right) \\
& =l(\mathbb{V}) \widetilde{\prod} \operatorname{length}\left(\operatorname{Edges}\left(\Delta_{\omega}\right)\right)\left(\operatorname{Trop}\left(\mathbb{G}^{e}\right) \cdot \operatorname{Trop}\left(\mathbb{L}_{\mathcal{B}}\right)\right)
\end{aligned}
$$

Thus we obtain

$$
\boldsymbol{m}_{\operatorname{Sev}(\Delta, \delta)}(\omega)=l(\mathbb{V}) \widetilde{\prod} \text { length }\left(\operatorname{Edges}\left(\Delta_{\omega}\right)\right)
$$

Now we consider the case that there is a non-primitive parallelogram in the subdivision $\Delta_{\omega}$. We need to consider a certain projection. Let us begin with a general setting. Let $\mathcal{S}(\Delta)$ be a nodal subdivision which may have non-primitive parallelograms. As we studied in $\$ 3.2$, in this case, the variety $\mathbb{V}_{\partial \mathcal{S}(\Delta), \text { nodal }}$ is contained in every coordinate hyperplane $H_{a}$ of the ambient projective space $\mathbb{P}_{\Delta}$ defined by $x_{a}=0$, where $a$ is a special point in a non-primitive parallelogram. In particular, $\mathbb{V}_{\partial \mathcal{S}(\Delta), \text { nodal }}$ is disjoint from the big open torus $\mathbb{T}_{\Delta}$. Let $H_{\mathcal{S}(\Delta)}$ be the intersection of all such coordinate hyperplanes $H_{a}$. Let $\mathbb{T}_{\mathcal{S}(\Delta)} \subset H_{\mathcal{S}(\Delta)}$ be the big open torus in $H_{\mathcal{S}(\Delta)}$ so that $\mathbb{V}_{\partial \mathcal{S}(\Delta), \text { nodal }} \subset \mathbb{T}_{\mathcal{S}(\Delta)}$. Let $\pi_{\mathcal{S}(\Delta)}$ be the projection from $\mathbb{P}_{\Delta}$ to $H_{\mathcal{S}(\Delta)}$,

$$
\pi_{\mathcal{S}(\Delta)}: \mathbb{P}_{\Delta} \rightarrow H_{\mathcal{S}(\Delta)}
$$

Now we consider the case when the subdivision $\mathcal{S}(\Delta)$ is given by a c-vector $\omega$ of $\operatorname{Sev}(\Delta, \delta)$ with maximal rank, that is, $\mathcal{S}(\Delta)=\Delta_{\omega}$ and $\operatorname{rank}(\omega)=\operatorname{dim}(\operatorname{Sev}(\Delta, \delta))$. We impose one more condition that $\omega$ is a regular point of the tropical Severi variety $\operatorname{Trop}(\operatorname{Sev}(\Delta, \delta))$, that is, $\operatorname{Trop}(\operatorname{Sev}(\Delta, \delta))$ coincides with an affine space of dimension $\operatorname{dim}(\operatorname{Sev}(\Delta, \delta))$ locally near $\omega$. (Warning: the maximality of rank of $\omega$ does not necessarily imply that $\omega$ is a regular point of $\operatorname{Trop}(\operatorname{Sev}(\Delta, \delta))$.) Then we obtain the following theorem.

Theorem 4.7. Let $\omega$ be a c-vector of $\operatorname{Sev}(\Delta, \delta)$. Suppose that $\omega$ satisfies the following conditions:

- The rank of $\omega$ is maximal, that is, $\operatorname{rank}(\omega)=\operatorname{dim}(\operatorname{Sev}(\Delta, \delta))$;

- $\omega$ is a regular point in $\operatorname{Trop}(\operatorname{Sev}(\Delta, \delta))$.

Then the following statements hold true:

(1) The projection $\pi_{\Delta_{\omega}}$ is a bijection from $\operatorname{in}_{\omega} \operatorname{Sev}(\Delta, \delta)$ to $\mathbb{V}=\mathbb{V}_{\partial \Delta_{\omega}, \text { nodal }}$.

(2) The initial scheme $\operatorname{in}_{\omega} \operatorname{Sev}(\Delta, \delta)$ is the union of finitely many translations of a torus $\mathbb{G}^{e *}$ of dimension $\operatorname{dim}(\operatorname{Sev}(\Delta, \delta))$ which is sent to $\mathbb{G}^{e}$ by the projection $\pi_{\Delta_{\omega}}$, where $\mathbb{G}^{e}$ is the identity component of $\mathbb{G}=\mathbb{G}_{\partial \Delta_{\omega}, \text { nodal }}$.

(3) The weight of $\omega$ on $\operatorname{in}_{\omega} \operatorname{Sev}(\Delta, \delta)$, that is, the number of such translations of the torus $\mathbb{G}^{\text {e* }}$ counted with multiplicity, is equal to

$$
\boldsymbol{m}_{\operatorname{Sev}(\Delta, \delta)}(\omega)=l(\mathbb{V}) \cdot \widetilde{\prod} \text { length }\left(\operatorname{Edges}\left(\Delta_{\omega}\right)\right),
$$

as defined in the previous theorem. 
Proof. The first and second statements are deduced straight-forwardly from the conditions on $\omega$. Let us show the last statement. It is a slight adjustment of the proof in the previous theorem adding the consideration of the projection $\pi_{\Delta_{w}}$. Shustin's first enumeration (4.1) deduces the following:

$$
\left(\operatorname{Trop}(\mathbb{V}) \pi_{*}\left(\operatorname{Trop}\left(\mathbb{L}_{\mathcal{B}}\right)\right)\right)=\frac{\prod 2 \operatorname{area}(\text { Triangles })}{\widetilde{\prod} \text { length }\left(\operatorname{Edges}\left(\Delta_{\omega}\right)\right)},
$$

where $\pi_{*}: \mathbb{R}^{n} \rightarrow \mathbb{R}^{|\mathcal{A}|}$ is the projection corresponding to $\pi=\pi_{\Delta_{\omega}}$. Moreover, from the second enumeration 4.2 , we obtain the following:

$$
\left(\operatorname{Trop}\left(\operatorname{in}_{\omega} \operatorname{Sev}(\Delta, \delta)\right) \cdot \operatorname{Trop}\left(\mathbb{L}_{\mathcal{B}}\right)\right)=\prod 2 \text { area }(\text { Triangles }) .
$$

Thus,

$(4.18)$

$$
\begin{aligned}
m_{\operatorname{Sev}(\Delta, \delta)}(\omega)\left(\operatorname{Trop}\left(\mathbb{G}^{e *}\right) \cdot \operatorname{Trop}\left(\mathbb{L}_{\mathcal{B}}\right)\right) & =\left(\operatorname{Trop}\left(\operatorname{in} \operatorname{sev}_{\operatorname{Sev}}(\Delta, \delta)\right) \cdot \operatorname{Trop}\left(\mathbb{L}_{\mathcal{B}}\right)\right) \\
& =\prod 2 \operatorname{area}(\operatorname{Triangles}) \\
& =\widetilde{\prod} \text { length }\left(\operatorname{Edges}\left(\Delta_{\omega}\right)\right) \cdot \frac{\prod \text { 2area }(\operatorname{Triangles})}{\widetilde{\prod} \text { length }\left(\operatorname{Edges}\left(\Delta_{\omega}\right)\right)} \\
& =\widetilde{\prod} \text { length }\left(\operatorname{Edges}\left(\Delta_{\omega}\right)\right)\left(\operatorname{Trop}(\mathbb{V}) \cdot \pi_{*}\left(\operatorname{Trop}\left(\mathbb{L}_{\mathcal{B}}\right)\right)\right) \\
& =l(\mathbb{V}) \widetilde{\prod} \operatorname{length}\left(\operatorname{Edges}\left(\Delta_{\omega}\right)\right)\left(\operatorname{Trop}\left(\mathbb{G}^{e}\right) \cdot \pi_{*}\left(\operatorname{Trop}\left(\mathbb{L}_{\mathcal{B}}\right)\right)\right) \\
& =l(\mathbb{V}) \widetilde{\prod} \text { length }\left(\operatorname{Edges}\left(\Delta_{\omega}\right)\right)\left(\operatorname{Trop}\left(\mathbb{G}^{e *}\right) \cdot \operatorname{Trop}\left(\mathbb{L}_{\mathcal{B}}\right)\right)
\end{aligned}
$$

The last equality can be seen easily by considering the projection $\pi_{*}$ as follows: by choosing a coordinate system, $\left(\operatorname{Trop}\left(\mathbb{G}^{e}\right) \cdot \pi_{*}\left(\operatorname{Trop}\left(\mathbb{L}_{\mathcal{B}}\right)\right)\right)$ is the determinant of a matrix ( $M_{1} \mid M_{2}$ ), where $M_{1}$ and $M_{2}$ are found from lattice bases of $\operatorname{Trop}\left(\mathbb{G}^{e}\right)$ and $\pi_{*}\left(\operatorname{Trop}\left(\mathbb{L}_{\mathcal{B}}\right)\right)$, respectively. Then $\left(\operatorname{Trop}\left(\mathbb{G}_{\circ}^{*}\right) \cdot \operatorname{Trop}\left(\mathbb{L}_{\mathcal{B}}\right)\right)$ is the determinant of a matrix of the form of $\left(\begin{array}{c|c|c}M_{1} & M_{2} & 0 \\ \hline * & 0 & I\end{array}\right)$, where $I$ is the identity matrix. Therefore $\left(\operatorname{Trop}\left(\mathbb{G}^{e}\right) \cdot \pi_{*}\left(\operatorname{Trop}\left(\mathbb{L}_{\mathcal{B}}\right)\right)\right)=\left(\operatorname{Trop}\left(\mathbb{G}^{e *}\right) \cdot \operatorname{Trop}\left(\mathbb{L}_{\mathcal{B}}\right)\right)$.

Thus,

$$
\boldsymbol{m}_{\operatorname{Sev}(\Delta, \delta)}(\omega)=l(\mathbb{V}) \widetilde{\prod} \text { length }\left(\operatorname{Edges}\left(\Delta_{\omega}\right)\right) .
$$

Remark 4.8. As a corollary, if $\operatorname{in}_{\omega} \operatorname{Sev}(\Delta, \delta) \neq \emptyset$ and the subdivision $\Delta_{\omega}$ is either simple or nodal but not both, then $\operatorname{rank}(\omega)<r$.

4.4. The degrees of Severi varieties. In this section, we study Mikhalkin's Correspondence theorem with respect to tropical intersection theory. Let us review this theorem.

Definition 4.9. 11, Definition 2.41]

(1) Let $\mathcal{S}(\Delta)$ be a subdivision of $\Delta$. We say that the distinct points $x_{1}, \ldots, x_{\zeta} \in$ $\mathbb{Q}^{2}$ are in $\mathcal{S}(\Delta)$-general position, if the condition for tropical curves to pass through $x_{1}, \ldots, x_{\zeta}$ ("base-point-condition") cuts out the tropical cone $\mathcal{T} C(\mathcal{S}(\Delta))$ either the empty set, or a polyhedron of codimension $\zeta$.

(2) We say that the distinct points $x_{1}, \ldots, x_{\zeta}$ are in $\Delta$-general position (or simply, generic points), if they are $\mathcal{S}(\Delta)$-general for all subdivisions $\mathcal{S}(\Delta)$ of $\Delta$.

Lemma 4.10. [11, Lemma 2.42] For any given convex lattice polygon $\Delta$, the set of $\Delta$-general configurations $x_{1}, \ldots, x_{\zeta}$ is dense in $\left(\mathbb{Q}^{2}\right)^{\zeta}$. 
To present the correspondence theorem we need one more numeric invariant assigned to a subdivision $\mathcal{S}(\Delta)$ of a polygon $\Delta$, besides the rank of $\mathcal{S}(\Delta)$ : suppose $\mathcal{S}(\Delta)$ is nodal, that is, the subpolygons are either triangles or parallelograms. Then the (Mikhalkin's) multiplicity of $\mathcal{S}(\Delta)$ is by definition

$$
\mu(\mathcal{S}(\Delta)):=\prod \text { 2area(Triangles), }
$$

the product of twice areas of all the triangles in $\mathcal{S}(\Delta)$.

Theorem 4.11 (Mikhalkin's Correspondence Theorem). [11, Theorem 2.43], [17]

Let $\mathcal{P}$ be a set of $r=\operatorname{dim}(\operatorname{Sev}(\Delta, \delta))$ points in $\mathbb{R}^{2}$ which are in $\Delta$-general position. Then

$$
\operatorname{degree}(\operatorname{Sev}(\Delta, \delta))=\sum_{\omega} \mu(\omega)
$$

where the sum runs over all tropical curves $\tau_{\omega}$ of degree $\Delta$ passing through all the points in $\mathcal{P}$. $\left(\mu(\omega)\right.$ is by definition $\mu\left(\Delta_{\omega}\right)$.)

First, we show that the set $\mathcal{S}$ of such tropical curves described above is in oneto-one correspondence with the set-theoretic transversal intersection of two tropical varieties (Definition 4.12), $\operatorname{Trop}(\operatorname{Sev}(\Delta, \delta)) \cap \operatorname{Trop}(\mathcal{L}(\boldsymbol{p}))$. Then, we show that Mikhalkin's multiplicity of any curve in the set $\mathcal{S}$ is equal to the tropical intersection multiplicity of the corresponding point in the intersection $\operatorname{Trop}(\operatorname{Sev}(\Delta, \delta)) \cap$ $\operatorname{Trop}(\mathcal{L}(\boldsymbol{p}))$. Thus, Mikhalkin's enumeration is equal to the computation of the degree $(\operatorname{Trop}(\operatorname{Sev}(\Delta, \delta)) \cdot \operatorname{Trop}(\mathcal{L}(\boldsymbol{p})))$. Let us begin with the definition of $\mathcal{L}(\boldsymbol{p})$.

Definition 4.12. Let $\boldsymbol{p}=\left\{p_{1}, \ldots, p_{\zeta}\right\} \subset\left(\mathbb{K}^{*}\right)^{2}$ be a finite set of points in $\left(\mathbb{K}^{*}\right)^{2}$. Define $\mathcal{L}(\boldsymbol{p}) \subset \mathbb{P}_{\Delta}(\mathbb{K})$ to be the parameter space of algebraic curves on the toric surface $X_{\Delta}(\mathbb{K})$ passing through all the points in $\boldsymbol{p}$. This parameter space $\mathcal{L}(\boldsymbol{p})$ is the complete intersection of hyperplanes $\mathcal{H}_{p_{j}} \subset \mathbb{P}_{\Delta}(\mathbb{K})$ defined by the condition of passing through the point $p_{j},(j=1, \ldots, \zeta)$. The intersection of $\mathcal{L}(\boldsymbol{p})$ with the big open torus $\mathbb{T}_{\Delta}$ is again denoted by $\mathcal{L}(\boldsymbol{p})$.

Lemma 4.13. An integral vector $\omega$ is a c-vector of the hyperplane $\mathcal{H}_{q}$, i.e., $\omega \in$ $\operatorname{Trop}\left(\mathcal{H}_{q}\right)$, if and only if the tropical curve $\tau_{\omega}$ passes through the point $\operatorname{Val}(q)=$ $\left(\operatorname{Val}\left(q_{1}\right), \operatorname{Val}\left(q_{2}\right)\right)$, where $q=\left(q_{1}, q_{2}\right) \in\left(\mathbb{K}^{*}\right)^{2}$.

Proof. Let $q_{1}=\alpha t^{m}+$ l.o.t. and $q_{2}=\beta t^{n}+$ l.o.t. and thus $\operatorname{Val}(q)=(m, n)$. Now $\mathcal{H}_{q}$ is the hyperplane defined by the linear polynomial

$$
\sum_{a=\left(a_{1}, a_{2}\right) \in \Delta \cap \mathbb{Z}^{2}}\left(\alpha t^{m}+\text { l.o.t. }\right)^{a_{1}}\left(\beta t^{n}+\text { l.o.t }\right)^{a_{2}} c_{a}=0
$$

in the variables $c_{a}, \quad a \in \Delta \cap \mathbb{Z}^{2}$. Thus, the support of $\operatorname{Trop}\left(\mathcal{H}_{q}\right)$ is the corner locus of the map:

$$
\left(x_{a}\right)_{a \in \Delta \cap \mathbb{Z}^{2}} \mapsto \max _{a \in \Delta \cap \mathbb{Z}^{2}}\left\{x_{a}+(m, n) \cdot\left(a_{1}, a_{2}\right)\right\}
$$

Also, the tropical curve $\tau_{\omega}$ is by definition the corner locus of the map:

$$
(X, Y) \mapsto \max _{a \in \Delta \cap \mathbb{Z}^{2}}\left\{\omega_{a}+(X, Y) \cdot\left(a_{1}, a_{2}\right)\right\}
$$

The statement follows in a straightforward way.

From the Lemma 4.13 above, we see that $\omega$ is a c-vector of $\mathcal{L}(\boldsymbol{p})$ if and only if the tropical curve $\tau_{\omega}$ passes through all the points $\operatorname{Val}\left(p_{1}\right), \ldots, \operatorname{Val}\left(p_{\zeta}\right)$ in $\mathbb{R}^{2}$. 
Theorem 4.14. Let $\boldsymbol{p}=\left\{p_{1}, \ldots, p_{r}\right\} \in\left(\left(\mathbb{K}^{*}\right)^{2}\right)^{r}$ be a configuration of $r$ generic points in $\left(\mathbb{K}^{*}\right)^{2}$ so that $\operatorname{Val}(\boldsymbol{p})=\left\{\operatorname{Val}\left(p_{1}\right), \ldots, \operatorname{Val}\left(p_{r}\right)\right\} \in\left(\mathbb{Q}^{2}\right)^{r}$ is in $\Delta$-general position, $\operatorname{Trop}(\mathcal{L}(\boldsymbol{p})) \cap \operatorname{Trop}(\operatorname{Sev}(\Delta, \delta))$ is a transversal intersection and the linear system 4.27 is independent, where $r=\operatorname{dim}(\operatorname{Sev}(\Delta, \delta))$. Then the following statements hold true:

(1) The intersection $\operatorname{Trop}(\mathcal{L}(\boldsymbol{p})) \cap \operatorname{Trop}(\operatorname{Sev}(\Delta, \delta))$ is in one-to-one correspondence with the set of tropical curves $\tau_{\omega}$ passing through all the points in $\operatorname{Val}(p)$.

(2) The extrinsic intersection multiplicity (\$2.4. 1) of $\operatorname{Trop}(\mathcal{L}(\boldsymbol{p}))$ and $\operatorname{Trop}(\operatorname{Sev}(\Delta, \delta))$ at $\omega \in \operatorname{Trop}(\mathcal{L}(\boldsymbol{p})) \cap \operatorname{Trop}(\operatorname{Sev}(\Delta, \delta))$ is

$$
\xi(\omega ; \operatorname{Trop}(\mathcal{L}(\boldsymbol{p})), \operatorname{Trop}(\operatorname{Sev}(\Delta, \delta)))=\frac{\prod \text { 2area }(\text { Triangles })}{l(\mathbb{V}) \cdot \widetilde{\prod} \text { length }(\text { Edges })} .
$$

(3) The tropical intersection multiplicity (§2.4. 2) of $\operatorname{Trop}(\mathcal{L}(\boldsymbol{p}))$ and $\operatorname{Trop}(\operatorname{Sev}(\Delta, \delta))$ at $\omega \in \operatorname{Trop}(\mathcal{L}(\boldsymbol{p})) \cap \operatorname{Trop}(\operatorname{Sev}(\Delta, \delta))$ is equal to Mikhalkin's multiplicity of the tropical curve $\tau_{\omega}$ :

$$
\boldsymbol{m}(\omega ; \operatorname{Trop}(\mathcal{L}(\boldsymbol{p})), \operatorname{Trop}(\operatorname{Sev}(\Delta, \delta)))=\prod \text { 2area(Triangles) } .
$$

Proof. Let us prove the first statement: Since the set of c-vectors are open dense in the tropicalization of a variety, we can assume that the intersection points in $\operatorname{Trop}(\mathcal{L}(\boldsymbol{p})) \cap \operatorname{Trop}(\operatorname{Sev}(\Delta, \delta))$ are all c-vectors, in particular, they are all rational vectors. From Lemma 4.13 and Shustin's combinatorial characterization Theorem 2.14 the first statement follows.

To prove the second statement, we find neighborhoods of $\omega$ in $\operatorname{Trop}(\operatorname{Sev}(\Delta, \delta))$ and in $\operatorname{Trop}(\mathcal{L}(\boldsymbol{p}))$. Then we compute the volume of the corresponding principal parallelepiped, which is by definition the extrinsic intersection multiplicity of $\operatorname{Trop}(\mathcal{L}(\boldsymbol{p}))$ and $\operatorname{Trop}(\operatorname{Sev}(\Delta, \delta))$ at $\omega(\S 2.4$. 1). Since the points are in $\Delta$-general position and the base-point-condition cuts out the cone $\mathcal{K}\left(\mathcal{T S}(\Delta)_{\omega}\right)$ non-empty $\left(c c(\omega)_{\mathbb{Z}} \in \mathcal{K}\left(\mathcal{T S}(\Delta)_{\omega}\right)\right)$, the rank of $\omega$ must be at least $r=\operatorname{dim}(\operatorname{Sev}(\Delta, \delta))$ and so is equal to $r$, since $\omega$ is a c-vector of $\operatorname{Sev}(\Delta, \delta)$. Also $\omega$ is a regular point of both $\operatorname{Trop}(\operatorname{Sev}(\Delta, \delta))$ and $\operatorname{Trop}(\mathcal{L}(\boldsymbol{p}))$, being a traversal-intersection-point. Thus, by Theorem 4.7. near $\omega$, the support of $\operatorname{Trop}(\operatorname{Sev}(\Delta, \delta))$ is equal to $\operatorname{Trop}\left(\mathbb{G}^{e *}\right)$, which is a $r$-dimensional linear space. Now let us consider a full-dimensional neighborhood of $\omega$ in $\operatorname{Trop}(\mathcal{L}(\boldsymbol{p}))$. The tropical curves corresponding to points in such neighborhood are tropical curves passing through the $r$ points $\operatorname{Val}\left(p_{1}\right), \ldots, \operatorname{Val}\left(p_{r}\right)$ and their degrees are subsets of $\Delta$. In particular, the tropical curve $\tau_{\omega}$ also passes through the points. Since $\operatorname{rank}(\omega)=r$, they lie on $r$ distinct edges of $\tau_{\omega}$ which correspond to some $r$ edges of the subdivision $\Delta_{\omega}$. If $\sigma_{i} \in \operatorname{Edges}\left(\Delta_{\omega}\right)$ correspond to a point $\operatorname{Val}\left(p_{i}\right)$ and $a_{i}, a_{i}^{\prime}$ are the endpoints of $\sigma_{i}, \quad 1 \leq i \leq r$, then we have the following linear conditions on $\omega\left(a_{i}\right)$ and $\omega\left(a_{i}^{\prime}\right)$ :

$$
\omega\left(a_{i}\right)-\omega\left(a_{i}^{\prime}\right)=\left(a_{i}^{\prime}-a_{i}\right) \cdot \operatorname{Val}\left(p_{i}\right), \quad(i=1, \ldots, r .)
$$

Let $\mathcal{B}$ be the set of vertices of $\Delta_{\omega}$ which are involved in this independent linear system. Thus the values of $\omega$ on $\mathcal{B}$ are fixed and ones on $\left(\Delta \cap \mathbb{Z}^{2}\right) \backslash \mathcal{B}$ can be any values. Thus we can see that near $\omega$, the support of $\operatorname{Trop}(\mathcal{L}(\boldsymbol{p}))$ is equal to $\operatorname{Trop}\left(\mathbb{L}_{\mathcal{B}}\right)$, where $\mathbb{L}_{\mathcal{B}}$ is defined in the proof of Theorem 4.6. Note that Trop $\left(\mathbb{G}^{e *}\right)$ and $\operatorname{Trop}\left(\mathbb{L}_{\mathcal{B}}\right)$ have constant weighting function 1 . Thus we can compute the extrinsic intersection 
multiplicity of $\operatorname{Trop}(\mathcal{L}(\boldsymbol{p}))$ and $\operatorname{Trop}(\operatorname{Sev}(\Delta, \delta))$ at $\omega$ as follows:

$$
\begin{aligned}
\xi(\omega ; \operatorname{Trop}(\mathcal{L}(\boldsymbol{p})), \operatorname{Trop}(\operatorname{Sev}(\Delta, \delta))) & =\operatorname{Trop}\left(\mathbb{G}^{e *}\right) \cdot \operatorname{Trop}\left(\mathbb{L}_{\mathcal{B}}\right) \\
& =\operatorname{Trop}\left(\mathbb{G}^{e}\right) \cdot \pi_{*}\left(\operatorname{Trop}\left(\mathbb{L}_{\mathcal{B}}\right)\right) \\
& =\frac{\operatorname{Trop}(\mathbb{V}) \cdot \pi_{*}\left(\operatorname{Trop}\left(\mathbb{L}_{\mathcal{B}}\right)\right)}{l(\mathbb{V})} \\
& =\frac{\prod 2 \operatorname{Trea}(\operatorname{Triangles})}{\prod \text { length }\left(\operatorname{Edges}\left(\Delta_{\omega}\right)\right) l(\mathbb{V})}
\end{aligned}
$$

Now we prove the last statement: it follows from the definition of $\boldsymbol{m}(\omega)$ given in $\$ 2.4$

$$
\begin{aligned}
& \boldsymbol{m}(\omega ; \operatorname{Trop}(\mathcal{L}(\boldsymbol{p})), \operatorname{Trop}(\operatorname{Sev}(\Delta, \delta))) \\
& =\boldsymbol{m}_{\mathcal{L}(\boldsymbol{p})}(\omega) \cdot \boldsymbol{m}_{\operatorname{Sev}(\Delta, \delta)}(\omega) \cdot \xi(\omega ; \operatorname{Trop}(\mathcal{L}(\boldsymbol{p})), \operatorname{Trop}(\operatorname{Sev}(\Delta, \delta))) \\
& =1 \cdot l(\mathbb{V}) \cdot \widetilde{\prod l} \text { length }(\text { Edges }) \cdot \frac{\prod \text { 2area(Triangles) }}{\Pi \text { length }\left(\operatorname{Edges}\left(\Delta_{\omega}\right)\right) l(\mathbb{V})} \\
& =\prod \text { 2area }(\text { Triangles })
\end{aligned}
$$

Therefore from the Theorem 4.14, Mikhalkin's enumeration of tropical curves is equal to the computation of the degree $(\operatorname{Trop}(\mathcal{L}(\boldsymbol{p}) \cdot \operatorname{Trop}(\operatorname{Sev}(\Delta, \delta)))$.

\section{REFERENCES}

[1] L. Allermann, J. Rau, First steps in tropical intersection theory. Math. Z. 264 (2010), no. 3, 633-670.

[2] D. Bernstein, The number of roots of a system of equations. Functional Analysis and its Applications 9 (1975) 183-185.

[3] R. Bieri, J. Groves, The geometry of the set of characters induced by valuations. J.Reine Angew. Math. 347 (1984), 168-195.

[4] M. Einsiedler, M. Kapranov, D. Lind, Non-Archimedean amoebas and tropical varieties. J. Reine Angew. Math. 601 (2006), 139-157

[5] D. Eisenbud, Commutative Algebra with a View Towards Algebraic Geometry. Graduate Texts in Mathematics, Springer, New York, 1995

[6] F. Enriques, Sui moduli d'una classe di superficie e sul teorema d'esistenza per funzioni algebriche di due variabilis. Atti Accad. Sci. Torino, 47, 1912.

[7] W. Fulton, Introduction to toric varieties. Ann. Math. Studies 131, Princeton Univ. Press, Princeton N.J., 1993.

[8] W. Fulton, B. Sturmfels, Intersection theory on toric varieties. Topology 36 (1997) 335-353.

[9] Andreas Gathmann, Hannah Markwig, The numbers of tropical plane curves through points in general position. J. Reine Angew. Math. 602 (2007), 155-177.

[10] I. M. Gelfand, M. M. Kapranov, A. V. Zelevinsky, Discriminants, Resultants, and Multidimensional Determinants. Birkhauser, Boston 1994.

[11] I. Itenberg, G. Mikhalkin, E. Shustin, Tropical algebraic geometry. Oberwolfach Seminars, 35 Birkhauser Verlag, Basel, 2007. viii+103 pp.

[12] E.Katz, A tropical toolkit. Expo. Math. 27 (2009), no. 1, 1-36.

[13] B. Ya.Kazarnovskii, Truncations of systems of equations, ideals and varieties. (Russian) Izv. Ross. Akad. Nauk Ser. Mat. 63 (1999), no. 3, 119-132; translation in Izv. Math. 63 (1999), no. 3, 535-547

[14] B. Ya.Kazarnovskii, c-fans and Newton polyhedra of algebraic varieties. (Russian) Izv. Ross. Akad. Nauk Ser. Mat. 67 (2003), no. 3, 23-44; translation in Izv. Math.67 (2003), no. 3, 439-460

[15] B. Ya.Kazarnovskii, Multiplicative intersection theory and complex tropical varieties. (Russian) Izv. Ross. Akad. Nauk Ser. Mat. 71 (2007), no. 4, 19-68; translation in Izv. Math. 71 (2007), no. 4, 673-720

[16] Diane Maclagan, Bernd Sturmfels, Introduction to Tropical Geometry. preprint (textbook in progress), 2009

[17] G. Mikhalkin, Enumerative tropical algebraic geometry in $\mathbb{R}^{2}$. J. Amer.Math. Soc. 18 (2005), 313-377. 
[18] G. Mikhalkin, Tropical Geometry and its applications. International Congress of Mathematicians. Vol. II, 827-852, Eur. Math. Soc., Zürich, 2006.

[19] Sam Payne, Fibers of tropicalization. Math. Z. 262 (2009), no. 2, 301-311

[20] F. Severi, Vorlesungen über Algebraische Geometrie. Teubner, Leipzig, 1921.

[21] E. Shustin, Gluing of singular and critical points. Topology 37 (1998), no. 1, 195.217.

[22] E. Shustin, A tropical approach to enumerative geometry. Algebra i Analiz 17 (2005), no. 2, 170.214 (English translation: St. Petersburg Math. J. 17 (2006), 343-375

[23] E. Shustin, Patchworking singular algebraic curves, non-Archimedean amoebas and enumerative geometry. Preprint arXiv: math.AG/0211278.

[24] B. Sturmfels, J. Tevelev, Elimination theory for tropical varieties. Math. Res. Lett. 15 (2008), no. $3,543-562$.

Department of Mathematics and Statistics, McMaster University, 1280 Main Street West, Hamilton, Ontario L8S4K1, Canada

E-mail address: jyang@math.mcmaster.ca 